\title{
LARGE DEVIATIONS AND SUPPORT RESULTS FOR NONLINEAR SCHRÖDINGER EQUATIONS WITH ADDITIVE NOISE AND APPLICATIONS
}

\author{
ÉRIC GAUTIER ${ }^{1,2}$
}

\begin{abstract}
Sample path large deviations for the laws of the solutions of stochastic nonlinear Schrödinger equations when the noise converges to zero are presented. The noise is a complex additive Gaussian noise. It is white in time and colored in space. The solutions may be global or blow-up in finite time, the two cases are distinguished. The results are stated in trajectory spaces endowed with topologies analogue to projective limit topologies. In this setting, the support of the law of the solution is also characterized. As a consequence, results on the law of the blow-up time and asymptotics when the noise converges to zero are obtained. An application to the transmission of solitary waves in fiber optics is also given.
\end{abstract}

Mathematics Subject Classification. 35Q51, 35Q55, 60F10, 60H15.

Received May 25, 2004. Revised December 15, 2004.

\section{INTRODUCTION}

In the present article, the stochastic nonlinear Schrödinger (NLS) equation with a power law nonlinearity and an additive noise is studied. The deterministic equation occurs as a basic model in many areas of physics: hydrodynamics, plasma physics, nonlinear optics, molecular biology. It describes the propagation of waves in media with both nonlinear and dispersive responses. It is an idealized model and does not take into account many aspects such as inhomogeneities, high order terms, thermal fluctuations, external forces which may be modeled as a random excitation (see $[11,14,16,17,20,21])$. Propagation in random media may also be considered. The resulting re-scaled equation is a random perturbation of the dynamical system of the following form:

$$
i \frac{\partial}{\partial t} \psi-\left(\Delta \psi+\lambda|\psi|^{2 \sigma} \psi\right)=\xi, \quad x \in \mathbb{R}^{d}, \quad t \geq 0, \quad \lambda= \pm 1,
$$

where $\xi$ is a complex valued space-time white noise with correlation function, following the notation used in $[16]$,

$$
\mathbb{E}\left[\xi\left(t_{1}, x_{1}\right) \bar{\xi}\left(t_{2}, x_{2}\right)\right]=D \delta_{t_{1}-t_{2}} \otimes \delta_{x_{1}-x_{2}}
$$

\footnotetext{
Keywords and phrases. Large deviations, stochastic partial differential equations, nonlinear Schrödinger equations, white noise, projective limit, support theorem, blow-up, solitary waves.

1 CREST-INSEE, URA D2200, 3 avenue Pierre Larousse, 92240 Malakoff, France.

2 IRMAR, UMR 6625, Université de Rennes 1, Campus de Beaulieu, 35042 Rennes Cedex, France;

eric.gautier@bretagne.ens-cachan.fr
}

(C) EDP Sciences, SMAI 2005 
$D$ is the noise intensity and $\delta$ denotes the Dirac mass. When $\lambda=1$ the nonlinearity is called focusing, otherwise it is defocusing.

With the notations of Section 2 , the unbounded operator $-i \Delta$ on $\mathrm{L}^{2}\left(\mathbb{R}^{d}\right)$ with domain $\mathrm{H}^{2}\left(\mathbb{R}^{d}\right)$ is skew-adjoint. Stone's theorem gives thus that it generates a unitary group $\left(S(t)=\mathrm{e}^{-i t \Delta}\right)_{t \in \mathbb{R}}$. The Fourier transform gives that this group is also unitary on every Sobolev space based on $\mathrm{L}^{2}\left(\mathbb{R}^{d}\right)$. Consequently, there is no smoothing effect in the Sobolev spaces. We are thus unable to treat the space-time white noise and will consider a complex valued centered Gaussian noise, white in time and colored in space.

In the present article, the formalism of stochastic evolution equations in Banach spaces as presented in [6] is adopted. This point of view is preferred to the field and martingale measure stochastic integral approach, see [23], in order to use a particular property of the group, namely hyper-contractivity. The Strichartz estimates, presented in the next section, show that some integrability property is gained through time integration and "convolution" with the group. In this setting, the Gaussian noise is defined as the time derivative in the sense of distributions of a $Q$-Wiener process $(W(t))_{t \in[0,+\infty)}$ on $\mathrm{H}^{1}\left(\mathbb{R}^{d}\right)$. Here $Q$ is the covariance operator of the law of the $\mathrm{H}^{1}\left(\mathbb{R}^{d}\right)$-random variable $W(1)$, which is a centered Gaussian measure. With the Itô notations, the stochastic evolution equation is written

$$
i \mathrm{~d} u-\left(\Delta u+\lambda|u|^{2 \sigma} u\right) \mathrm{d} t=\mathrm{d} W
$$

The initial datum $u_{0}$ is a function of $\mathrm{H}^{1}\left(\mathbb{R}^{d}\right)$. We will consider solutions of NLS that are weak solutions in the sense used in the analysis of partial differential equations or equivalently mild solutions which satisfy

$$
u(t)=S(t) u_{0}-i \lambda \int_{0}^{t} S(t-s)\left(|u(s)|^{2 \sigma} u(s)\right) \mathrm{d} s-i \int_{0}^{t} S(t-s) \mathrm{d} W(s) .
$$

The well posedness of the Cauchy problem associated to (1.1) in the deterministic case depends on the size of $\sigma$. If $\sigma<\frac{2}{d}$, the nonlinearity is subcritical and the Cauchy problem is globally well posed in $\mathrm{L}^{2}\left(\mathbb{R}^{d}\right)$ or $\mathrm{H}^{1}\left(\mathbb{R}^{d}\right)$. If $\sigma=\frac{2}{d}$, critical nonlinearity, or $\frac{2}{d}<\sigma<\frac{2}{d-2}$ when $d \geq 3$ or simply $\sigma>\frac{2}{d}$ otherwise, supercritical nonlinearity, the Cauchy problem is locally well posed in $\mathrm{H}^{1}\left(\mathbb{R}^{d}\right)$, see [19]. In this latter case, if the nonlinearity is defocusing, the solution is global. In the focusing case some initial data yield global solutions while it is known that other initial data yield solutions which blow up in finite time, see [5,22].

In [7], the $\mathrm{H}^{1}\left(\mathbb{R}^{d}\right)$ results have been generalized to the stochastic case and existence and uniqueness results are obtained for the stochastic equation under the same conditions on $\sigma$. Continuity with respect to the initial data and the perturbation is proved. It is shown that the proof of global existence for a defocusing nonlinearity or for a focusing nonlinearity with a subcritical exponent, could be adapted in the stochastic case even if the mass

and Hamiltonian

$$
M(u(t))=\|u(t)\|_{\mathrm{L}^{2}\left(\mathbb{R}^{d}\right)}^{2}
$$

$$
H(u(t))=\frac{1}{2} \int_{\mathbb{R}^{d}}|\nabla u(t)|^{2} \mathrm{~d} x-\frac{\lambda}{2 \sigma+2} \int_{\mathbb{R}^{d}}|u(t)|^{2 \sigma+2} \mathrm{~d} x
$$

are no longer conserved. For a focusing nonlinearity and critical or supercritical exponents, the solution may blow-up in finite time. The blow-up time is denoted by $\tau(\omega)$. It satisfies either $\lim _{t \rightarrow \tau(\omega)}\|u(t)\|_{\mathrm{H}^{1}\left(\mathbb{R}^{d}\right)}=+\infty$ or $\tau(\omega)=+\infty$, even if the solution is obtained by a fixed point argument in a ball of a space of more regular functions than $\mathrm{C}\left([0, T] ; \mathrm{H}^{1}\left(\mathbb{R}^{d}\right)\right)$.

In this article, we are interested in the law of the paths of the random solution. When the noise converges to zero, continuity with respect to the perturbation gives that the law converges to the Dirac mass on the deterministic solution. In the following, a large deviation result is shown. It gives the rate of convergence to zero, on an exponential scale, of the probability that paths are in sets that do not contain the deterministic solution. A general result is stated for the case where blow-up in finite time is possible and a second one for the particular case where the solutions are global. Also, the stronger the topology, the sharper are the estimates. We will therefore take advantage of the variety of spaces that can be considered for the fixed point argument, 
due to the integrability property, and present the large deviation principles in trajectory spaces endowed with projective limit topologies. A characterization of the support of the law of the solution in these trajectory spaces is proved. The two results can be transferred to weaker topologies or more generally by any continuous mapping. The first application is a proof that, for certain noises, with positive probability some solutions blow up after any time $T$. Some estimates on the law of the blow-up time when the noise converges to zero are also obtained. This study is yet another contribution to the study of the influence of a noise on the blow-up of the solutions of the focusing supercritical NLS, see in the case of an additive noise [8,9]. A second application is given. It consists in obtaining similar results as in [16] with an approach based on large deviations. The aim is to compute estimates of error probability in signal transmission in optical fibers when the medium is random and nonlinear, for small noises. Uniform large deviations for small noise asymptotics when the noise enters linearly as a random potential the NLS equation are studied in [18]. In that case we had to use a more elaborate proof based on the Freidlin and Wentzell inequality and the continuity of the skeleton with respect to the control on the level sets of the rate function of the initial Wiener process since in that case the Itô map fails to be continuous at the level of paths for the topologies we consider.

Section 2 is devoted to notations and properties of the group, of the noise and of the stochastic convolution. An extension of the result of continuity with respect to the stochastic convolution presented in [7] is also given. In Section 3, the large deviation principles (LDP) is presented. Section 4 is devoted to the support result and the two last sections to the applications.

\section{Notations AND PRELIMINARY RESUlts}

Throughout the paper the following notations will be used.

The set of positive integers and positive real numbers are denoted respectively by $\mathbb{N}^{*}$ and $\mathbb{R}_{+}^{*}$, while the set of real numbers different from 0 is denoted by $\mathbb{R}^{*}$.

For $p$ in $\mathbb{N}^{*}, \mathrm{~L}^{p}\left(\mathbb{R}^{d}\right)$ is the classical Lebesgue space of complex valued functions and $\mathrm{W}^{1, p}\left(\mathbb{R}^{d}\right)$ is the associated Sobolev space of $\mathrm{L}^{p}\left(\mathbb{R}^{d}\right)$ functions with first order derivatives, in the sense of distributions, in $\mathrm{L}^{p}\left(\mathbb{R}^{d}\right)$. When $p=2, \mathrm{H}^{s}\left(\mathbb{R}^{d}\right)$ denotes the fractional Sobolev space of tempered distributions $v \in \mathcal{S}^{\prime}\left(\mathbb{R}^{d}\right)$ such that the Fourier transform $\hat{v}$ satisfies $\left(1+|\xi|^{2}\right)^{s / 2} \hat{v} \in \mathrm{L}^{2}\left(\mathbb{R}^{d}\right)$. The space $\mathrm{L}^{2}\left(\mathbb{R}^{d}\right)$ is endowed with the inner product defined by $(u, v)_{\mathrm{L}^{2}\left(\mathbb{R}^{d}\right)}=\Re \int_{\mathbb{R}^{d}} u(x) \bar{v}(x) \mathrm{d} x$. Also, when it is clear that $\mu$ is a Borel measure on a specified Banach space, we simply write $\mathrm{L}^{2}(\mu)$ and do not specify the Banach space and Borel $\sigma$-field.

If $I$ is an interval of $\mathbb{R},\left(E,\|\cdot\|_{E}\right)$ a Banach space and $r$ belongs to $[1,+\infty]$, then $\mathrm{L}^{r}(I ; E)$ is the space of strongly Lebesgue measurable functions $f$ from $I$ into $E$ such that $t \rightarrow\|f(t)\|_{E}$ is in $\operatorname{L}^{r}(I)$. Let $\mathrm{L}_{l o c}^{r}(0,+\infty ; E)$ be the respective spaces of locally integrable functions on $(0,+\infty)$. They are endowed with topologies of Fréchet space. The spaces $\mathrm{L}^{r}(\Omega ; E)$ are defined similarly.

We recall that a pair $(r, p)$ of positive numbers is called an admissible pair if $p$ satisfies $2 \leq p<\frac{2 d}{d-2}$ when $d>2(2 \leq p<+\infty$ when $d=2$ and $2 \leq p \leq+\infty$ when $d=1)$ and $r$ is such that $\frac{2}{r}=d\left(\frac{1}{2}-\frac{1}{p}\right)$. For example $(+\infty, 2)$ is an admissible pair.

When $E$ is a Banach space, we will denote by $E^{*}$ its topological dual space. For $x^{*} \in E^{*}$ and $x \in E$, the duality will be denoted $\left\langle x^{*}, x\right\rangle_{E^{*}, E}$.

We recall that $\Phi$ is a Hilbert Schmidt operator from a Hilbert space $H$ into a Hilbert space $\tilde{H}$ if it is a linear continuous operator such that, given a complete orthonormal system $\left(e_{j}^{H}\right)_{j \in \mathbb{N}}$ of $H, \sum_{j \in \mathbb{N}}\left\|\Phi e_{j}^{H}\right\|_{\tilde{H}}^{2}<+\infty$. We will denote by $\mathcal{L}_{2}(H, \tilde{H})$ the space of Hilbert Schmidt operators from $H$ into $\tilde{H}$ endowed with the norm

$$
\|\Phi\|_{\mathcal{L}_{2}(H, \tilde{H})}=\operatorname{tr}\left(\Phi \Phi^{*}\right)=\sum_{j \in \mathbb{N}}\left\|\Phi e_{j}^{H}\right\|_{\tilde{H}}^{2},
$$

where $\Phi^{*}$ denotes the adjoint of $\Phi$ and $\operatorname{tr}$ the trace. We denote by $\mathcal{L}_{2}^{s, r}$ the corresponding space for $H=\mathrm{H}^{s}\left(\mathbb{R}^{d}\right)$ and $\tilde{H}=\mathrm{H}^{r}\left(\mathbb{R}^{d}\right)$. In the introduction $\Phi$ has been taken in $\mathcal{L}_{2}^{0,1}$. 
When $A$ and $B$ are two Banach spaces, $A \cap B$, where the norm of an element is defined as the maximum of the norm in $A$ and in $B$, is a Banach space. The following Banach spaces defined for the admissible pair $(r(p), p)$ and positive $T$ by

$$
X^{(T, p)}=\mathrm{C}\left([0, T] ; \mathrm{H}^{1}\left(\mathbb{R}^{d}\right)\right) \cap \mathrm{L}^{r(p)}\left(0, T ; \mathrm{W}^{1, p}\left(\mathbb{R}^{d}\right)\right)
$$

will be of particular interest.

The probability space will be denoted by $(\Omega, \mathcal{F}, \mathbb{P})$. Also, $x \wedge y$ stands for the minimum of the two real numbers $x$ and $y$ and $x \vee y$ for the maximum. We recall that a rate function $I$ is a lower semicontinuous function and that a good rate function $I$ is a rate function such that for every $c>0,\{x: I(x) \leq c\}$ is a compact set. Finally, we will denote by supp $\mu$ the support of a probability measure $\mu$ on a topological vector space. It is the complement of the largest open set of null measure.

\subsection{Properties of the group}

When the group acts on the Schwartz space $\mathcal{S}\left(\mathbb{R}^{d}\right)$, the Fourier transform gives the following analytic expression

$$
\forall u_{0} \in \mathcal{S}\left(\mathbb{R}^{d}\right), \forall t \neq 0, S(t) u_{0}=\frac{1}{(4 i \pi t)^{\frac{d}{2}}} \int_{\mathbb{R}^{d}} \mathrm{e}^{-i \frac{|x-y|^{2}}{4 t}} u_{0}(y) \mathrm{d} y .
$$

The Fourier transform also gives that the adjoint of $S(t)$ in $\mathrm{L}^{2}\left(\mathbb{R}^{d}\right)$ and in every Sobolev space on $\mathrm{L}^{2}\left(\mathbb{R}^{d}\right)$ is $S(-t)$, the same bounded operator with time reversal.

The Strichartz estimates, see [19], are the following

i) $\forall u_{0} \in \mathrm{L}^{2}\left(\mathbb{R}^{d}\right), \forall(r, p)$ admissible pair,

$$
t \mapsto S(t) u_{0} \in \mathrm{C}\left(\mathbb{R} ; \mathrm{L}^{2}\left(\mathbb{R}^{d}\right)\right) \cap \mathrm{L}^{r}\left(\mathbb{R} ; \mathrm{L}^{p}\left(\mathbb{R}^{d}\right)\right)
$$

and there exists a positive constant $c$ such that,

$$
\left\|S(\cdot) u_{0}\right\|_{\mathrm{L}^{r}\left(\mathbb{R} ; \mathrm{L}^{p}\left(\mathbb{R}^{d}\right)\right)} \leq c\left\|u_{0}\right\|_{\mathrm{L}^{2}\left(\mathbb{R}^{d}\right)}
$$

ii / For $T>0$, for all $(r(p), p)$ and $(r(q), q)$ two admissible pairs, if $s$ and $\rho$ are the conjugate exponents of $r(q)$ and $q$, i.e. $\frac{1}{s}+\frac{1}{r(q)}=1$ and $\frac{1}{q}+\frac{1}{\rho}=1$,

$$
\forall f \in \mathrm{L}^{s}\left(0, T ; \mathrm{L}^{\rho}\left(\mathbb{R}^{d}\right)\right), \Lambda f \in \mathrm{C}\left([0, T] ; \mathrm{L}^{2}\left(\mathbb{R}^{d}\right)\right) \cap \mathrm{L}^{r(p)}\left(0, T ; \mathrm{L}^{p}\left(\mathbb{R}^{d}\right)\right)
$$

where $\Lambda$ is defined by $\Lambda f=\int_{0}^{\cdot} S(\cdot-s) f(s) \mathrm{d} s$. Moreover, $\Lambda$ is a continuous linear operator from $\mathrm{L}^{s}\left(0, T ; \mathrm{L}^{\rho}\left(\mathbb{R}^{d}\right)\right)$ into $\mathrm{L}^{r(p)}\left(0, T ; \mathrm{L}^{p}\left(\mathbb{R}^{d}\right)\right)$ with a norm that does not depend on $T$.

Remark 2.1. The first estimate gives the integrability property of the group, the second gives the integrability of the convolution that allows to treat the nonlinearity.

\subsection{Topology and trajectory spaces}

Let us introduce a topological space that allows us to treat the subcritical case or the defocusing case. When $d>2$, we set

$$
\mathcal{X}_{\infty}=\bigcap_{T \in \mathbb{R}_{+}^{*}, 2 \leq p<\frac{2 d}{d-2}} X^{(T, p)}
$$

it is endowed with the projective limit topology, see $[3,13]$. When $d=2$ and $d=1$ we write $p \in[2,+\infty)$.

The set of indices $\left(\mathbb{R}_{+}^{*} \times\left[2, \frac{2}{d-2}\right), \prec\right)$ when $d>2$ or $\left(\mathbb{R}_{+}^{*} \times[2,+\infty), \prec\right)$ when $d=2$ or $d=1$, where $(T, p) \prec(S, q)$ if $T \leq S$ and $p \leq q$, is a partially ordered right-filtering set. 
If $(T, p) \prec(S, q)$ and $u \in X^{(S, q)}$, Hölder's inequality gives that for $\alpha$ such that $\frac{1}{p}=\frac{\alpha}{q}+\frac{1-\alpha}{2}$,

$$
\|u(t)\|_{\mathrm{L}^{p}\left(\mathbb{R}^{d}\right)} \leq\|u(t)\|_{\mathrm{L}^{2}\left(\mathbb{R}^{d}\right)}^{1-\alpha}\|u(t)\|_{\mathrm{L}^{q}\left(\mathbb{R}^{d}\right)}^{\alpha} .
$$

Consequently,

$$
\|u(t)\|_{\mathrm{W}^{1, p}\left(\mathbb{R}^{d}\right)} \leq(d+1)\|u(t)\|_{\mathrm{H}^{1}\left(\mathbb{R}^{d}\right)}^{1-\alpha}\|u(t)\|_{\mathrm{W}^{1, q}\left(\mathbb{R}^{d}\right)}^{\alpha} .
$$

By time integration, along with Hölder's inequality, the fact that $r(q)=\alpha r(p)$ and that $T \leq S$, we obtain that $u$ is a function of $X^{(T, p)}$ and

$$
\|u\|_{X^{(T, p)}} \leq(d+1)\|u\|_{X^{(S, q)}} .
$$

If we denote by $p_{(S, q)}^{(T, p)}$ the dense and continuous embeddings from $X^{(S, q)}$ into $X^{(T, p)}$, they satisfy the consistency conditions

$$
\forall(T, p) \prec(S, q) \prec(R, r), p_{(R, r)}^{(T, p)}=p_{(R, r)}^{(S, q)} \circ p_{(S, q)}^{(T, p)} .
$$

Consequently, the projective limit topology is well defined by the following neighborhood basis, given for $\varphi_{1}$ in $\mathcal{X}_{\infty}$ by

$$
U\left(\varphi_{1} ;(T, p) ; \epsilon\right)=\left\{\varphi \in \bigcap_{\left(T^{\prime}, p^{\prime}\right) \in J} X^{\left(T^{\prime}, p^{\prime}\right)}:\left\|\varphi-\varphi_{1}\right\|_{X^{(T, p)}}<\epsilon\right\} .
$$

It is the weakest topology on the intersection such that for every $(T, p) \in J$, the injection $p_{(T, p)}: \mathcal{X}_{\infty} \rightarrow X^{(T, p)}$ is continuous. It is a standard fact, see [3], that $\mathcal{X}_{\infty}$ is a Hausdorff topological space.

Following from (2.1), a countable neighborhood basis of $\varphi_{1}$ is given by $\left(U\left(\varphi_{1} ;(n, p(l)) ; \frac{1}{k}\right)\right)_{(n, k, l) \in\left(N^{*}\right)^{3}}$, where $p(l)=2+\frac{4}{d-2}-\frac{1}{l}$ and $l>\frac{d-2}{4}$ if $d>2$. If $d=2$ and $d=1$, we take $p(l)=l$.

Also it is convenient, for measurability issues, to note that $\mathcal{X}_{\infty}$ can be turned into a complete separable metric space, i.e. a Polish space, setting

$$
\forall(x, y) \in \mathcal{X}_{\infty}^{2}, d(x, y)=\sum_{n>\frac{d-2}{4}} \frac{1}{2^{n}}\left(\|x-y\|_{X^{(n, p(n))}} \wedge 1\right) .
$$

It can be checked that it is also a locally convex Fréchet space.

The following spaces are introduced for the case where blow-up may occur. Adding a point $\Delta$ to the space $\mathrm{H}^{1}\left(\mathbb{R}^{d}\right)$ and adapting slightly the proof of Alexandroff's compactification, it can be seen that the open sets of $\mathrm{H}^{1}\left(\mathbb{R}^{d}\right)$ and the complement in $\mathrm{H}^{1}\left(\mathbb{R}^{d}\right) \cup\{\Delta\}$ of the closed bounded sets of $\mathrm{H}^{1}\left(\mathbb{R}^{d}\right)$ define the open sets of a topology on $\mathrm{H}^{1}\left(\mathbb{R}^{d}\right) \cup\{\Delta\}$. This topology induces on $\mathrm{H}^{1}\left(\mathbb{R}^{d}\right)$ the topology of $\mathrm{H}^{1}\left(\mathbb{R}^{d}\right)$. Also, with such a topology $\mathrm{H}^{1}\left(\mathbb{R}^{d}\right) \cup\{\Delta\}$ is a Hausdorff topological space. Note that in [1], where diffusions are studied, the compactification of $\mathbb{R}^{d}$ is considered. Nonetheless, compactness is not an important feature and the above construction is enough for the following.

The space $\mathrm{C}\left([0,+\infty) ; \mathrm{H}^{1}\left(\mathbb{R}^{d}\right) \cup\{\Delta\}\right)$ is the space of continuous functions with value in $\mathrm{H}^{1}\left(\mathbb{R}^{d}\right) \cup\{\Delta\}$. Also, if $f$ belongs to $\mathrm{C}\left([0,+\infty) ; \mathrm{H}^{1}\left(\mathbb{R}^{d}\right) \cup\{\Delta\}\right)$ we denote the blow-up time by

$$
\mathcal{T}(f)=\inf \{t \in[0,+\infty): f(t)=\Delta\} .
$$

As in [1], a space of exploding paths, where $\Delta$ acts as a cemetery, is introduced. We set

$$
\mathcal{E}\left(\mathrm{H}^{1}\left(\mathbb{R}^{d}\right)\right)=\left\{f \in \mathrm{C}\left([0,+\infty) ; \mathrm{H}^{1}\left(\mathbb{R}^{d}\right) \cup\{\Delta\}\right): f\left(t_{0}\right)=\Delta \Rightarrow \forall t \geq t_{0}, f(t)=\Delta\right\} .
$$

It is endowed with the topology defined by the following neighborhood basis given for $\varphi_{1}$ in $\mathcal{E}\left(\mathrm{H}^{1}\left(\mathbb{R}^{d}\right)\right)$ by

$$
V_{T, \epsilon}\left(\varphi_{1}\right)=\left\{\varphi \in \mathcal{E}\left(\mathrm{H}^{1}\left(\mathbb{R}^{d}\right)\right): \mathcal{T}(\varphi) \geq T,\left\|\varphi_{1}-\varphi\right\|_{\mathrm{L}^{\infty}\left([0, T] ; \mathrm{H}^{1}\left(\mathbb{R}^{d}\right)\right)} \leq \epsilon\right\}
$$

where $T<\mathcal{T}\left(\varphi_{1}\right)$ and $\epsilon>0$. 
As a consequence of the topology of $\mathcal{E}\left(\mathrm{H}^{1}\left(\mathbb{R}^{d}\right)\right)$, the function $\mathcal{T}: \mathcal{E}\left(\mathrm{H}^{1}\left(\mathbb{R}^{d}\right)\right) \rightarrow[0,+\infty]$ is sequentially lower semicontinuous, this is to say that if a sequence of functions $\left(f_{n}\right)_{n \in \mathbb{N}}$ converges to $f$ then $\varliminf_{n \rightarrow+\infty} \mathcal{T}\left(f_{n}\right) \geq \mathcal{T}(f)$. Following from (2.1), the topology of $\mathcal{E}\left(\mathrm{H}^{1}\left(\mathbb{R}^{d}\right)\right)$ is also defined by the countable neighborhood basis given for $\varphi_{1} \in \mathcal{E}\left(\mathrm{H}^{1}\left(\mathbb{R}^{d}\right)\right)$ by $\left(V_{\mathcal{T}\left(\varphi_{1}\right)-\frac{1}{n}, \frac{1}{k}}\left(\varphi_{1}\right)\right)_{(n, k) \in\left(\mathbb{N}^{*}\right)^{2}}$. Therefore $\mathcal{T}$ is a lower semicontinuous mapping.

Note that, as topological spaces, the two following spaces satisfy the identity

$$
\left\{f \in \mathcal{E}\left(\mathrm{H}^{1}\left(\mathbb{R}^{d}\right)\right): \mathcal{T}(f)=+\infty\right\}=\mathrm{C}\left([0,+\infty) ; \mathrm{H}^{1}\left(\mathbb{R}^{d}\right)\right) .
$$

Finally, the analogue of the intersection in the subcritical case endowed with projective limit topology is defined, when $d>2$, by

$$
\mathcal{E}_{\infty}=\left\{f \in \mathcal{E}\left(\mathrm{H}^{1}\left(\mathbb{R}^{d}\right)\right): \forall p \in\left[2, \frac{2 d}{d-2}\right), \forall T \in[0, \mathcal{T}(f)), f \in \mathrm{L}^{r(p)}\left(0, T ; \mathrm{W}^{1, p}\left(\mathbb{R}^{d}\right)\right)\right\} .
$$

When $d=2$ and $d=1$ we write $p \in[2,+\infty)$. It is endowed with the topology defined for $\varphi_{1}$ in $\mathcal{E}_{\infty}$ by the following neighborhood basis

$$
W_{T, p, \epsilon}\left(\varphi_{1}\right)=\left\{\varphi \in \mathcal{E}_{\infty}: \mathcal{T}(\varphi) \geq T,\left\|\varphi_{1}-\varphi\right\|_{X^{(T, p)}} \leq \epsilon\right\} .
$$

where $T<\mathcal{T}\left(\varphi_{1}\right), p$ is as above and $\epsilon>0$. From the same arguments as for the space $\mathcal{X}_{\infty}, \mathcal{E}_{\infty}$ is a Hausdorff topological space. Also, as previously, (2.1) gives that the topology can be defined for $\varphi_{1}$ in $\mathcal{E}_{\infty}$ by the countable neighborhood basis $\left(W_{\mathcal{T}\left(\varphi_{1}\right)-\frac{1}{n}, p(n), \frac{1}{k}}\left(\varphi_{1}\right)\right)_{(n, k) \in\left(\mathbb{N}^{*}\right)^{2}: n>\frac{d-2}{4}}$.

If we denote again by $\mathcal{T}: \mathcal{E}_{\infty} \rightarrow[0,+\infty]$ the blow-up time, since $\mathcal{E}_{\infty}$ is continuously embedded into $\mathcal{E}\left(\mathrm{H}^{1}\left(\mathbb{R}^{d}\right)\right)$, $\mathcal{T}$ is lower semicontinuous. Thus, since $\{[0, t], t \in[0,+\infty]\}$ is a $\pi$-system that generates the Borel $\sigma$-algebra of $[0,+\infty], \mathcal{T}$ is measurable. Note also that, as topological spaces, the following spaces are identical

$$
\left\{f \in \mathcal{E}_{\infty}: \mathcal{T}(f)=+\infty\right\}=\mathcal{X}_{\infty} .
$$

\subsection{Statistical properties of the noise}

The $Q$-Wiener process $W$ is such that its trajectories are in $\mathrm{C}\left([0,+\infty) ; \mathrm{H}^{1}\left(\mathbb{R}^{d}\right)\right)$. We assume in the following that $Q=\Phi \Phi^{*}$ where $\Phi$ is a Hilbert-Schmidt operator from $\mathrm{L}^{2}\left(\mathbb{R}^{d}\right)$ into $\mathrm{H}^{1}\left(\mathbb{R}^{d}\right)$. The Wiener process can therefore be written as $W=\Phi W_{c}$ where $W_{c}$ is a cylindrical Wiener process.

We recall that for any orthonormal basis $\left(e_{j}\right)_{j \in \mathbb{N}}$ of $\mathrm{L}^{2}\left(\mathbb{R}^{d}\right)$, there exists a sequence of real independent Brownian motions $\left(\beta_{j}\right)_{j \in \mathbb{N}}$ such that $W_{c}=\sum_{j \in \mathbb{N}} \beta_{j} e_{j}$. The sum $W_{c}=\sum_{j \in \mathbb{N}} \beta_{j} e_{j}$ is well defined in every Hilbert space $H$ such that $\mathrm{L}^{2}\left(\mathbb{R}^{d}\right)$ is embedded into $H$ with a Hilbert Schmidt embedding. We say that it is cylindrical because it is such that the decomposition of $W_{c}(1)$ on cylinder sets $\left(e_{1}, \ldots, e_{N}\right)$ are the finite dimensional centered Gaussian variables $\left(\beta_{1}(1), \ldots, \beta_{N}(1)\right)$ with a covariance equal to the identity. The law of $W(1)$ is thus the direct image measure by the Hilbert-Schmidt mapping $\Phi$ of the natural extension of the corresponding sequence of centered Gaussian measures in finite dimensions, with a covariance equal to identity. In other words it is the bona-fide $\sigma$-additive direct image measure of a Gaussian cylindrical measure. Also, formally, for $T$ positive the coefficients of the series expansion of the derivative of $W_{c}$ on the tensor product of the complete orthonormal systems of $\mathrm{L}^{2}\left(\mathbb{R}^{d}\right)$ and of $\mathrm{L}^{2}(0, T)$, given for example by the time derivative of the eigenvectors of the correlation operator of the law on $\mathrm{C}([0, T])$ of the Brownian motions, is a sequence of independent real-valued standard normal random variables. It is thus a Gaussian white noise.

In reference [16] the authors define the correlation function by the quantity

$$
\mathbb{E}\left[\frac{\partial}{\partial t} W_{c}(t+s, x+z) \overline{\frac{\partial}{\partial t} W_{c}(t, x)}\right]
$$


writing down formally the series expansion we obtain in the case of the white noise $2 \delta_{0}(s) \otimes \delta_{0}(z)$. In the case of our space-colored noise, we obtain the multiplication of $\delta_{0}(s)$ by the $\mathrm{L}^{2}\left(\mathbb{R}^{d}\right)$ function $\sum_{j \in \mathbb{N}} \Phi e_{j}(x+z) \overline{\Phi e_{j}(x)}$, where $\left(e_{j}\right)_{j \in \mathbb{N}}$ is a complete orthonormal system of $\mathrm{L}^{2}\left(\mathbb{R}^{d}\right)$. Also as the operator $\Phi$ belongs to $\mathcal{L}_{2}^{0,1}$ and thus to $\mathcal{L}_{2}^{0,0}$ it may be defined through the kernel $\mathcal{K}(x, y)=\frac{1}{2} \sum_{j \in \mathbb{N}} \Phi \overline{e_{j}}(x) e_{j}(y)$ of $\mathrm{L}^{2}\left(\mathbb{R}^{d} \times \mathbb{R}^{d}\right)$, considering that $\left(e_{j}\right)_{j \in \mathbb{N}}$ consists of $\left(f_{j}\right)_{j \in \mathbb{N}}$ a complete orthonormal system of $\mathrm{L}^{2}\left(\mathbb{R}^{d}, \mathbb{R}\right)$ and of $\left(i f_{j}\right)_{j \in \mathbb{N}}$. This means that for any square integrable function $u, \Phi u(x)=\int_{\mathbb{R}^{d}} \mathcal{K}(x, y) u(y) \mathrm{d} y$. In that case we could write the correlation function

$$
\left(2 \int_{\mathbb{R}^{d}} \mathcal{K}(x+z, u) \overline{\mathcal{K}}(x, u) \mathrm{d} u\right) \delta_{0}(s) .
$$

In the following we assume that the probability space is endowed with the filtration $\mathcal{F}_{t}=\mathcal{N} \cup \sigma\left\{W_{s}, 0 \leq s \leq t\right\}$ where $\mathcal{N}$ denotes the $\mathbb{P}$-null sets.

\subsection{The random perturbation}

We define the stochastic convolution by $Z(t)=\int_{0}^{t} S(t-s) \mathrm{d} W(s)$ and the operator $\mathcal{L}$ on $\mathrm{L}^{2}\left(0, T ; \mathrm{L}^{2}\left(\mathbb{R}^{d}\right)\right)$ by

$$
\mathcal{L} h(t)=\int_{0}^{t} I \circ S(t-s) \Phi h(s) \mathrm{d} s, h \in \mathrm{L}^{2}\left(0, T ; \mathrm{L}^{2}\left(\mathbb{R}^{d}\right)\right),
$$

where $I$ is the injection of $\mathrm{H}^{1}\left(\mathbb{R}^{d}\right)$ into $\mathrm{L}^{2}\left(\mathbb{R}^{d}\right)$.

Proposition 2.2. The stochastic convolution defines a measurable mapping from $(\Omega, \mathcal{F})$ into $\left(\mathcal{X}_{\infty}, \mathcal{B}^{X}\right)$, where $\mathcal{B}^{X}$ stands for the Borel $\sigma$-field. Its law is denoted by $\mu^{Z}$.

The direct images $\mu^{Z ;(T, p)}=p_{(T, p) *} \mu^{Z}$ on the real Banach spaces $X^{(T, p)}$ are centered Gaussian measures of reproducing kernel Hilbert space $(R K H S) H_{\mu^{Z ;(T, p)}}=\operatorname{im} \mathcal{L}$ with the norm of the image structure.

Proof. Setting $F(t)=\int_{0}^{t} S(-u) \mathrm{d} W(u)$, for $t \in \mathbb{R}^{+}, Z(t)=S(t) F(t)$ follows. Indeed, if $\left(f_{j}\right)_{j \in \mathbb{N}}$ is a complete orthonormal system of $\mathrm{H}^{1}\left(\mathbb{R}^{d}\right)$, a straightforward calculation gives that $\left(Z(t), f_{j}\right)_{\mathrm{H}^{1}\left(\mathbb{R}^{d}\right)}=\left(S(t) F(t), f_{j}\right)_{\mathrm{H}^{1}\left(\mathbb{R}^{d}\right)}$ for every $j$ in $\mathbb{N}$. The continuity of the paths in $\mathrm{H}^{1}\left(\mathbb{R}^{d}\right)$ follows from the construction of the stochastic integral with respect to the Wiener process since the deterministic operator integrand satisfies $\int_{0}^{T}\|S(-u) \Phi\|_{\mathcal{L}_{2}^{0,1}}^{2}<+\infty$ and from the strong continuity of the group.

Step 1. We claim that the mapping $Z$ is measurable from $(\Omega, \mathcal{F})$ into $\left(X^{(T, p)}, \mathcal{B}^{(T, p)}\right)$, where $\mathcal{B}^{(T, p)}$ denotes the associated Borel $\sigma$-field.

Since $X^{(T, p)}$ is a Polish space, every open set is a countable union of open balls and consequently $\mathcal{B}^{(T, p)}$ is generated by open balls. Note that the event $\left\{\omega \in \Omega:\|Z(\omega)-x\|_{X^{(T, p)}} \leq r\right\}$ is equal to

$$
\left(\bigcap_{s \in \mathbb{Q} \cap[0, T]}\left\{\omega \in \Omega:\|Z(s)(\omega)-x\|_{\mathrm{H}^{1}\left(\mathbb{R}^{d}\right)} \leq r\right\}\right) \bigcap\left\{\omega \in \Omega:\|Z(\omega)-x\|_{\mathrm{L}^{r(p)}\left(0, T ; \mathrm{W}^{1, p}\left(\mathbb{R}^{d}\right)\right)} \leq r\right\} .
$$

Also, note that, since $(Z(t))_{t \in \mathbb{R}^{+}}$is a collection of $\mathrm{H}^{1}\left(\mathbb{R}^{d}\right)$ random variables, the first part is a countable intersection of elements of $\mathcal{F}$. Consequently, it suffices to show that $: \omega \mapsto(t \mapsto Z(t))$ defines a $L^{r(p)}\left(0, T ; \mathrm{W}^{1, p}\left(\mathbb{R}^{d}\right)\right)$ random variable.

Consider $\left(\Phi_{n}\right)_{n \in \mathbb{N}}$ a sequence of operators of $\mathcal{L}_{2}^{0,2}$ converging to $\Phi$ for the topology of $\mathcal{L}_{2}^{0,1}$ and $Z_{n}$ the associated stochastic convolutions. The Sobolev injections along with Hölder's inequality give that when $d>2$ and $2 \leq p \leq \frac{2 d}{d-2}, \mathrm{H}^{1}\left(\mathbb{R}^{d}\right)$ is continuously embedded in $\mathrm{L}^{p}\left(\mathbb{R}^{d}\right)$. It also gives that, when $d=2, \mathrm{H}^{1}\left(\mathbb{R}^{d}\right)$ is continuously embedded in every $\mathrm{L}^{p}\left(\mathbb{R}^{d}\right)$ for every $p \in[2,+\infty)$ and for every $p \in[2,+\infty]$ when $d=1$. Consequently, for every $n$ in $\mathbb{N}, Z_{n}$ defines a $\mathrm{C}\left([0, T] ; \mathrm{H}^{2}\left(\mathbb{R}^{d}\right)\right)$ random variable and therefore a $\mathrm{L}^{r(p)}\left(0, T ; \mathrm{W}^{1, p}\left(\mathbb{R}^{d}\right)\right)$ random variable for the corresponding values of $p$. 
Revisiting the proof of Proposition 3.1 in reference [7] and letting $2 \sigma+2$ be replaced by any of the previous values of $p$ besides $p=+\infty$ when $d=1$, the necessary measurability issues to apply the Fubini's theorem are satisfied. Also, one gets the same estimates and that there exists a constant $C(d, p)$ such that for every $n$ and $m$ in $\mathbb{N}$,

$$
\mathbb{E}\left[\left\|Z_{n+m}(\omega)-Z_{n}(\omega)\right\|_{L^{r(p)}\left(0, T ; \mathrm{W}^{1, p}\left(\mathbb{R}^{d}\right)\right)}^{r}\right] \leq C(d, p) T^{\frac{r}{2}-1}\left\|\Phi_{n+m}-\Phi_{n}\right\|_{\mathcal{L}_{2}^{0,1}}^{r}
$$

The sequence $\left(Z_{n}\right)_{n \in \mathbb{N}}$ is thus a Cauchy sequence of the Banach space $\mathrm{L}^{r}\left(\Omega ; \mathrm{L}^{r}\left(0, T ; \mathrm{W}^{1, p}\left(\mathbb{R}^{d}\right)\right)\right)$ and converges to $\tilde{Z}$. The previous calculation also gives that

$$
\mathbb{E}\left[\left\|Z_{n}(\omega)-Z(\omega)\right\|_{L^{r(p)}\left(0, T ; \mathrm{L}^{p}\left(\mathbb{R}^{d}\right)\right)}^{r}\right] \leq C(d, p) T^{\frac{r}{2}-1}\left\|\Phi_{n}-\Phi\right\|_{\mathcal{L}_{2}^{0,1}}^{r}
$$

Therefore $\tilde{Z}=Z, Z$ belongs to $\mathrm{L}^{r(p)}\left(0, T ; \mathrm{W}^{1, p}\left(\mathbb{R}^{d}\right)\right)$ and it defines a measurable mapping as expected.

Note that in $\mathcal{X}_{\infty}$, to simplify the notations, we did not write the cases $p=+\infty$ when $d=1$ or $p=\frac{2 d}{d-2}$ when $d>2$. We are indeed interested in results on the laws of the solutions of stochastic NLS and not really on the stochastic convolution. Also, the result of continuity in the next section shows that we necessarily lose on $p$ in order to interpolate with $2<p<p^{\prime}$ and have a nonzero exponent on the $\mathrm{L}^{2}\left(\mathbb{R}^{d}\right)$-norm. Therefore, even if it seems at first glance that we lose on the Sobolev's injections, it is not a restriction.

Step 2. We show that the mapping $Z$ is measurable with values in $\mathcal{X}_{\infty}$ with the Borel $\sigma$-field $\mathcal{B}^{\mathcal{X}_{\infty}}$.

From step 1 , given $x \in \mathcal{X}_{\infty}$, for every $n$ in $\mathbb{N}^{*}$ such that $n>\frac{d-2}{4}$ the mapping $\omega \mapsto\|Z(\omega)-x\|_{X^{(n, p(n))}}$ from $(\Omega, \mathcal{F})$ into $\left(\mathbb{R}^{+}, \mathcal{B}\left(\mathbb{R}^{+}\right)\right)$, where $\mathcal{B}\left(\mathbb{R}^{+}\right)$stands for the Borel $\sigma$-field of $\mathbb{R}^{+}$, is measurable. Thus

$$
\omega \mapsto d(Z(\omega), x)=\lim _{N \rightarrow+\infty} \sum_{n=1}^{N} \frac{1}{2^{n}}\left(\|Z(\omega)-x\|_{X^{(n, p(n))}} \wedge 1\right)
$$

is measurable. Consequently, for every $r$ in $\mathbb{R}^{+},\{\omega \in \Omega: d(Z(\omega), x)<r\}$ belongs to $\mathcal{F}$.

Note that the law $\mu^{Z ; \mathcal{X}_{\infty}}$ of $Z$ on the metric space $\mathcal{X}_{\infty}$, which is a positive Borel measure, is therefore also regular and consequently it is a Radon measure.

Step 3 (statements on the measures $\left.\mu^{Z ;(T, p)}\right)$. For $(T, p)$ in $\mathbb{R}_{+}^{*} \times\left[2, \frac{2}{d-2}\right)$ when $d>2$ or $\mathbb{R}_{+}^{*} \times[2,+\infty)$ when $d=2$ or $d=1$, let $i_{(T, p)}$ denote the continuous injections from $X^{(T, p)}$ into $\mathrm{L}^{2}\left(0, T ; \mathrm{L}^{2}\left(\mathbb{R}^{d}\right)\right)$ and $\mu^{Z ; L}=\left(i_{(T, p)}\right)_{*} \mu^{Z ;(T, p)}$. The $\sigma$-field on $\mathrm{L}^{2}\left(0, T ; \mathrm{L}^{2}\left(\mathbb{R}^{d}\right)\right)$ is the Borel $\sigma$-field. Let $h \in \mathrm{L}^{2}\left(0, T ; L^{2}\left(\mathbb{R}^{d}\right)\right)$, then

$$
\left(h, i_{(T, p)}(Z)\right)_{\mathrm{L}^{2}\left(0, T ; \mathrm{L}^{2}\left(\mathbb{R}^{d}\right)\right)}=\int_{0}^{T} \sum_{i, j=1}^{+\infty} \int_{0}^{t}\left(e_{j}, S(t-s) \Phi e_{i}\right)_{\mathrm{L}^{2}\left(\mathbb{R}^{d}\right)} \mathrm{d} \beta_{i}(s)\left(h(t), e_{j}\right)_{\mathrm{L}^{2}\left(\mathbb{R}^{d}\right)}
$$

and from classical computation it is the almost sure limit of a sum of independent centered Gaussian random variables, thus $\mu^{Z ; L}$ is a centered Gaussian measure.

Every linear continuous functional on $\mathrm{L}^{2}\left(0, T ; \mathrm{L}^{2}\left(\mathbb{R}^{d}\right)\right)$ defines by restriction a linear continuous functional on $X^{(T, p)}$. Thus, $\mathrm{L}^{2}\left(0, T ; \mathrm{L}^{2}\left(\mathbb{R}^{d}\right)\right)^{*}$ could be thought of as a subset of $\left(X^{(T, p)}\right)^{*}$. Since $i_{(T, p)}$ is a continuous injection, $\mathrm{L}^{2}\left(0, T ; \mathrm{L}^{2}\left(\mathbb{R}^{d}\right)\right)^{*}$ is dense in $\left(X^{(T, p)}\right)^{*}$ for the weak topology $\sigma\left(\left(X^{(T, p)}\right)^{*}, X^{(T, p)}\right)$. This means that, given $x^{*} \in\left(X^{(T, p)}\right)^{*}$, there exists a sequence $\left(h_{n}\right)_{n \in \mathbb{N}}$ of elements of $\mathrm{L}^{2}\left(0, T ; \mathrm{L}^{2}\left(\mathbb{R}^{d}\right)\right)$ such that for every $x \in X^{(T, p)}$,

$$
\lim _{n \rightarrow+\infty}\left(h_{n}, i_{(T, p)}(x)\right)_{\mathrm{L}^{2}\left(0, T ; \mathrm{L}^{2}\left(\mathbb{R}^{d}\right)\right)}=\left\langle x^{*}, x\right\rangle_{\left(X^{(T, p)}\right)^{*}, X^{(T, p)}} .
$$

In other words, the random variable $\left\langle x^{*}, \cdot\right\rangle_{\left(X^{(T, p)}\right)^{*}, X^{(T, p)}}$ is a pointwise limit of $\left(h_{n}, i_{(T, p)}(\cdot)\right)_{\mathrm{L}^{2}\left(0, T ; \mathrm{L}^{2}\left(\mathbb{R}^{d}\right)\right)}$ which are, from the above, centered Gaussian random variables. As a consequence, $\mu^{Z ;(T, p)}$ is a centered Gaussian measure. 
Recall that the RKHS $H_{\mu z ; L}$ of $\mu^{Z ; L}$ is im $R^{L}$ where $R^{L}$ is the mapping from $H_{\mu^{Z ; L}}^{*}=\overline{\mathrm{L}^{2}\left(0, T ; \mathrm{L}^{2}\left(\mathbb{R}^{d}\right)\right)^{*}} \mathrm{~L}^{2}\left(\mu^{Z ; L}\right)$ with the inner product derived from the one in $\mathrm{L}^{2}\left(\mu^{Z ; L}\right)$ into $\mathrm{L}^{2}\left(0, T ; \mathrm{L}^{2}\left(\mathbb{R}^{d}\right)\right)$ defined for $\varphi$ in $H_{\mu z, L}^{*}$ by

$$
R^{L}(\varphi)=\int_{\mathrm{L}^{2}\left(0, T ; \mathrm{L}^{2}\left(\mathbb{R}^{d}\right)\right)} x \varphi(x) \mu^{Z ; L}(\mathrm{~d} x)
$$

The same is true for $H_{\mu^{Z ;(T, p)}}$ replacing $\mathrm{L}^{2}\left(0, T ; \mathrm{L}^{2}\left(\mathbb{R}^{d}\right)\right)$ by $X^{(T, p)}$ and $\mu^{Z ; L}$ by $\mu^{Z ;(T, p)}$.

Since $\mu^{Z ; L}$ is the image of $\mu^{Z ;(T, p)}$, taking $x^{*} \in \mathrm{L}^{2}\left(0, T ; \mathrm{L}^{2}\left(\mathbb{R}^{d}\right)\right)^{*}$, we obtain that

$$
\begin{aligned}
\left\|x^{*}\right\|_{\mathrm{L}^{2}\left(\mu^{Z ; L}\right)} & =\int_{\mathrm{L}^{2}\left(0, T ; \mathrm{L}^{2}\left(\mathbb{R}^{d}\right)\right)}<x^{*}, x>_{\mathrm{L}^{2}\left(0, T ; \mathrm{L}^{2}\left(\mathbb{R}^{d}\right)\right)^{*}, \mathrm{~L}^{2}\left(0, T ; \mathrm{L}^{2}\left(\mathbb{R}^{d}\right)\right)}^{2} \mu^{Z ; L}(\mathrm{~d} x) \\
& =\int_{X^{(T, p)}}<x^{*}, x>_{\mathrm{L}^{2}\left(0, T ; \mathrm{L}^{2}\left(\mathbb{R}^{d}\right)\right)^{*}, \mathrm{~L}^{2}\left(0, T ; \mathrm{L}^{2}\left(\mathbb{R}^{d}\right)\right)}^{2} \mu^{Z ;(T, p)}(\mathrm{d} x) \\
& =\int_{X^{(T, p)}}<x^{*}, x>_{\left(X^{(T, p)}\right)^{*}, X^{(T, p)}}^{2} \mu^{Z ;(T, p)}(\mathrm{d} x)=\left\|x^{*}\right\|_{\mathrm{L}^{2}\left(\mu^{Z ;(T, p)}\right)} .
\end{aligned}
$$

Therefore, from Lebesgue's dominated convergence theorem, we obtain that

$$
\left(X^{(T, p)}\right)^{*}=\overline{\mathrm{L}^{2}\left(0, T ; \mathrm{L}^{2}\left(\mathbb{R}^{d}\right)\right)^{*}} \sigma\left(\left(X^{(T, p)}\right)^{*}, X^{(T, p)}\right) \subset{\overline{\mathrm{L}^{2}\left(0, T ; \mathrm{L}^{2}\left(\mathbb{R}^{d}\right)\right)^{*}}}^{\mathrm{L}^{2}\left(\mu^{Z ;(T, p)}\right)}=H_{\mu^{Z ; L}}^{*}
$$

It follows that $H_{\mu^{Z ;(T, p)}}^{*} \subset H_{\mu^{Z ; L}}^{*}$.

The reverse inclusion follows from the fact that $\mathrm{L}^{2}\left(0, T ; \mathrm{L}^{2}\left(\mathbb{R}^{d}\right)\right)^{*} \subset\left(X^{(T, p)}\right)^{*}$.

The conclusion follows from the quite standard fact that the RKHS of $\mu^{Z ; L}$, which is a centered Gaussian measure on a Hilbert space, is equal to im $\mathcal{Q}^{\frac{1}{2}}$, with the norm of the image structure. $\mathcal{Q}$ denotes the covariance operator of the centered Gaussian measure, it is given, see [6], for $h \in \mathrm{L}^{2}\left(0, T ; \mathrm{L}^{2}\left(\mathbb{R}^{d}\right)\right)$, by

$$
\mathcal{Q} h(v)=\int_{0}^{T} \int_{0}^{u \wedge v} I S(v-s) \Phi \Phi^{*} S(s-u) I^{*} h(u) \mathrm{d} s \mathrm{~d} u .
$$

Corollary B.5 of reference [6] finally gives that $\operatorname{im} \mathcal{L}=\operatorname{im} \mathcal{Q}^{\frac{1}{2}}$.

\subsection{Continuity with respect to the perturbation}

Recall that the mild solution of stochastic NLS (1.3) could be written as a function of the perturbation. Let $v(x)$ denotes the solution of

$$
\left\{\begin{array}{l}
i \frac{\mathrm{d}}{\mathrm{d} t} v-\left(\Delta v+|v-i x|^{2 \sigma}(v-i x)\right)=0 \\
v(0)=u_{0}
\end{array}\right.
$$

or equivalently a fixed point of the functional

$$
\mathcal{F}_{x}(v)(t)=S(t) u_{0}-i \lambda \int_{0}^{t} S(t-s)\left(|(v-i x)(s)|^{2 \sigma}(v-i x)(s)\right) \mathrm{d} s,
$$

where $x$ is an element of $X^{(T, p)}, p$ is such that $p \geq 2 \sigma+2$ and $(T, p)$ is an arbitrary pair in $\mathbb{R}_{+}^{*} \times\left[2, \frac{2}{d-2}\right)$ when $d>2$ or $\mathbb{R}_{+}^{*} \times[2,+\infty)$ when $d=2$ or $d=1$. 
If $u$ is such that $u=v(Z)-i Z$ where $Z$ is the stochastic convolution, note that its regularity is given in the previous section, then $u$ is a solution of (1.3). Consequently, if $\mathcal{G}$ denotes the mapping that satisfies $\mathcal{G}(x)=v(x)-i x$ we obtain that $u=\mathcal{G}(Z)$.

The local existence follows from the fact that for $R>0$ and $r>0$ fixed, taking $\|x\|_{X^{(T, 2 \sigma+2)}} \leq R$ and $\left\|u_{0}\right\|_{\mathrm{H}^{1}\left(\mathbb{R}^{d}\right)} \leq r$, there exists a sufficiently small $T_{2 \sigma+2}^{*}$ such that the closed ball centered at 0 of radius $2 r$ is invariant and $\mathcal{F}_{x}$ is a contraction for the topology of $\mathrm{L}^{\infty}\left(\left[0, T_{2 \sigma+2}^{*}\right] ; \mathrm{L}^{2}\left(\mathbb{R}^{d}\right)\right) \cap \mathrm{L}^{r}\left(0, T_{2 \sigma+2}^{*} ; \mathrm{L}^{p}\left(\mathbb{R}^{d}\right)\right)$. Note that a closed ball of $X^{\left(T_{2 \sigma+2}^{*}, 2 \sigma+2\right)}$ is complete for the topology of $\mathrm{L}^{\infty}\left(\left[0, T_{2 \sigma+2}^{*}\right] ; \mathrm{L}^{2}\left(\mathbb{R}^{d}\right)\right) \cap \mathrm{L}^{r}\left(0, T_{2 \sigma+2}^{*} ; \mathrm{L}^{p}\left(\mathbb{R}^{d}\right)\right)$. The proof uses extensively the Strichartz' estimates, see [7] for a detailed proof. The same fixed point argument can be used for $\|x\|_{X^{(T, p)}} \leq R$ in a closed ball of radius $2 r$ in $X^{\left(T_{p}^{*}, p\right)}$ for every $T_{p}^{*}$ sufficiently small and $p \geq 2 \sigma+2$ such that $\left(T_{p}^{*}, p\right) \in J$. From $(2.1)$, there exists a unique maximal solution $v(x)$ that belongs to $\mathcal{E}_{\infty}$.

It could be deduced from Proposition 3.5 of [7], that the mapping $\mathcal{G}$ from $\mathcal{X}_{\infty}$ into $\mathcal{E}_{\infty}$ is a continuous mapping from $\bigcap_{T \in \mathbb{R}_{+}^{*}} X^{(T, 2 \sigma+2)}$ with the projective limit topology into $\mathcal{E}\left(\mathrm{H}^{1}\left(\mathbb{R}^{d}\right)\right)$. The result can be strengthened as follows.

Proposition 2.3. The mapping $\mathcal{G}$ from $\mathcal{X}_{\infty}$ into $\mathcal{E}_{\infty}$ is continuous.

Proof. Let $\tilde{x}$ be a function of $\mathcal{X}_{\infty}$ and $T<\mathcal{T}(\tilde{Z})$. Revisiting the proof of Proposition 3.5 of [7] and taking $\epsilon>0, p^{\prime} \geq 2 \sigma+2, R=1+\|\tilde{x}\|_{X^{\left(T, p^{\prime}\right)}}, r=1+\|v(\tilde{x})\|_{\mathrm{C}\left([0, T] ; \mathrm{H}^{1}\left(\mathbb{R}^{d}\right)\right)}$, and $2<p<p^{\prime}$, there exists $\eta>0$ satisfying $\eta<\frac{\epsilon}{2(d+1)} \wedge 1$ such that

$$
\forall x \in \mathcal{X}_{\infty}:\|x-\tilde{x}\|_{X^{\left(T, p^{\prime}\right)}} \leq \eta,\|v(x)-v(\tilde{x})\|_{\mathrm{C}\left([0, T] ; \mathrm{H}^{1}\left(\mathbb{R}^{d}\right)\right)} \leq\left(\frac{\epsilon}{2(d+1)(4 r)^{\alpha}}\right)^{\frac{1}{1-\alpha}} \wedge 1
$$

The constant $\alpha$ is the one that appears in the application of Hölder's inequality before (2.1). Consequently, since $v(x)$ and $v(\tilde{x})$ are functions of the closed ball centered at 0 and of radius $2 r$ in $X^{(T, p)}$, the triangle inequality gives that

$$
\|v(x)-v(\tilde{x})\|_{X^{\left(T, p^{\prime}\right)}} \leq 4 r
$$

The application of both Hölder's inequality and the triangle inequality allow to conclude that

$$
\forall x \in \mathcal{X}_{\infty}:\|x-\tilde{x}\|_{X^{\left(T, p^{\prime}\right)}} \leq \eta,\|\mathcal{G}(x)-\mathcal{G}(\tilde{x})\|_{X^{(T, p)}} \leq \epsilon
$$

which, from the definition of the neighborhood basis of $\mathcal{E}_{\infty}$, gives the continuity.

The following corollary is a consequence of the last statement of Section 2.2.

Corollary 2.4. In the focusing subcritical case or in the defocusing case, $\mathcal{G}$ is a continuous mapping from $\mathcal{X}_{\infty}$ into $\mathcal{X}_{\infty}$

The continuity allows us to define the law of the solutions of the stochastic NLS equations on $\mathcal{E}_{\infty}$ and in the cases of global existence in $\mathcal{X}_{\infty}$ as the direct image $\mu^{u}=\mathcal{G}_{*} \mu^{Z}$, the same notation will be used in both cases. Let consider the solutions of

$$
i \mathrm{~d} u_{\epsilon}-\left(\Delta u_{\epsilon}+\lambda\left|u_{\epsilon}\right|^{2 \sigma} u_{\epsilon}\right) \mathrm{d} t=\sqrt{\epsilon} \mathrm{d} W
$$

where $\epsilon \geq 0$. The laws of the solutions $u_{\epsilon}$ in the corresponding trajectory spaces are denoted by $\mu^{u_{\epsilon}}$, or equivalently $\mathcal{G}_{*} \mu^{Z_{\epsilon}}$ where $\mu^{Z_{\epsilon}}$ is the direct image of $\mu^{Z}$ under the transformation $x \mapsto \sqrt{\epsilon} x$ on $\mathcal{X}_{\infty}$. The continuity also gives that the family converges weakly to the Dirac mass on the deterministic solution $u_{d}$ as $\epsilon$ converges to zero. Next section is devoted to the study of the convergence towards 0 of rare events or tail events of the law of the solution $u_{\epsilon}$, namely large deviations. It allows to describe more precisely the convergence towards the deterministic measure. 


\section{SAMPlE PATH LARGE DEVIATIONS}

Theorem 3.1. The family of probability measures $\left(\mu^{u_{\epsilon}}\right)_{\epsilon \geq 0}$ on $\mathcal{E}_{\infty}$ satisfies a LDP of speed $\epsilon$ and good rate function

$$
I(u)=\frac{1}{2} \inf _{h \in \mathrm{L}^{2}\left(0,+\infty ; \mathrm{L}^{2}\left(\mathbb{R}^{d}\right)\right): \mathbf{S}(h)=u}\left\{\|h\|_{\mathrm{L}^{2}\left(0,+\infty ; \mathrm{L}^{2}\left(\mathbb{R}^{d}\right)\right)}^{2}\right\},
$$

where $\inf \emptyset=+\infty$ and $\mathbf{S}(h)$, called the skeleton, is the unique mild solution of the following control problem:

$$
\left\{\begin{array}{l}
i \frac{\mathrm{d}}{\mathrm{d} t} u=\Delta u+\lambda|u|^{2 \sigma} u+\Phi h \\
u(0)=u_{0} \in \mathrm{H}^{1}\left(\mathbb{R}^{d}\right) .
\end{array}\right.
$$

This is to say that for every Borel set $A$ of $\mathcal{E}_{\infty}$,

$$
-\inf _{u \in A} I(u) \leq \underline{\lim }_{\epsilon \rightarrow 0} \epsilon \log \mu^{u_{\epsilon}}(A) \leq \varlimsup_{\epsilon \rightarrow 0} \epsilon \log \mu^{u_{\epsilon}}(A) \leq-\inf _{u \in \bar{A}} I(u)
$$

The same result holds in $\mathcal{X}_{\infty}$ for the family of laws of the solutions in the cases of global existence.

Proof. The general LDP for centered Gaussian measures on real Banach spaces, see [12], gives that for a given pair $(T, p)$ in $\mathbb{R}_{+}^{*} \times\left[2, \frac{2}{d-2}\right)$ when $d>2$ or $\mathbb{R}_{+}^{*} \times[2,+\infty)$ when $d=2$ or $d=1$, the family $\left(p_{(T, p) *} \mu^{Z_{\epsilon}}\right)_{\epsilon \geq 0}$ satisfies a LDP on $X^{(T, p)}$ of speed $\epsilon$ and good rate function defined for $x \in X^{(T, p)}$ by,

$$
I^{Z ;(T, p)}(x)= \begin{cases}\frac{1}{2}\|x\|_{H_{\mu} Z ;(T, p)}^{2}, & \text { if } x \in H_{\mu} Z ;(T, p), \\ +\infty, & \text { otherwise }\end{cases}
$$

which, using Proposition 2.2, is equal to

$$
I^{Z ;(T, p)}(x)= \begin{cases}\frac{1}{2}\|x\|_{\text {im } \mathcal{L}}^{2}, & \text { if } x \in \operatorname{im~} \mathcal{L}, \\ +\infty, & \text { otherwise }\end{cases}
$$

Dawson-Gärtner's theorem, see [13], along with the monotone convergence theorem, allows us to deduce that the family $\left(\mu^{Z_{\epsilon}}\right)_{\epsilon \geq 0}$ satisfies the LDP with the good rate function defined for $x \in \mathcal{X}_{\infty}$ by

$$
\begin{aligned}
I^{Z}(x) & =\sup _{(T, p) \in J}\left\{I^{Z ;(T, p)}(x)\right\} \\
& =\left\{\begin{array}{c}
\sup _{(T, p) \in J}\left\{\frac{1}{2}\left\|\left(\Phi_{\mid \operatorname{ker} \Phi^{\perp}}\right)^{-1}\left(\frac{\mathrm{d}}{\mathrm{d} t} x+i \Delta x\right)\right\|_{\mathrm{L}^{2}\left(0, T ; \mathrm{L}^{2}\left(\mathbb{R}^{d}\right)\right)}^{2}\right\} \\
+\infty \quad \text { if } \frac{\mathrm{d}}{\mathrm{d} t} x+i \Delta x \notin \operatorname{im} \Phi
\end{array}\right. \\
= & \frac{1}{2} \inf _{h \in \mathrm{L}^{2}\left(0,+\infty ; \mathrm{L}^{2}\left(\mathbb{R}^{d}\right)\right): \mathcal{L}(h)=x}\left\{\|h\|_{\mathrm{L}^{2}\left(0,+\infty ; \mathrm{L}^{2}\left(\mathbb{R}^{d}\right)\right)}^{2}\right\} .
\end{aligned}
$$

It has been shown in Sections 2.2 and 2.5 that $\mathcal{G}$ is a continuous function from a Hausdorff topological space into another Hausdorff topological space. Consequently, both results follow from Varadhan's contraction principle along with the fact that if $\mathcal{G} \circ \mathcal{L}(h)=x$ then $x$ is the unique mild solution of the control problem (i.e. $x=\mathbf{S}(h))$. 
Remark 3.2. The rate function can be written

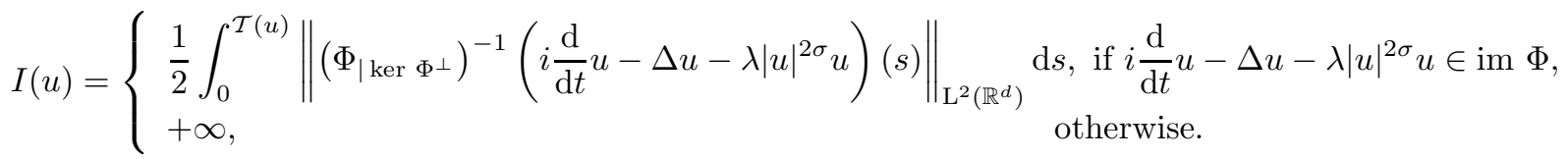

Remark 3.3. In the cases where blow-up may occur, the argument that will follow allows us to prove the weaker result that, given an $(T, p)$ in $\mathbb{R}_{+}^{*} \times\left[2, \frac{2}{d-2}\right)$ when $d>2$ or $\mathbb{R}_{+}^{*} \times[2,+\infty)$ when $d=2$ or $d=1$ and

$$
I^{(T, p)}(u)=\frac{1}{2} \inf _{h \in \mathrm{L}^{2}\left(0, T ; \mathrm{L}^{2}\left(\mathbb{R}^{d}\right)\right): \mathbf{S}(h)=u}\left\{\|h\|_{\mathrm{L}^{2}\left(0, T ; \mathrm{L}^{2}\left(\mathbb{R}^{d}\right)\right)}^{2}\right\},
$$

then for every bounded Borel set $A$ of $X^{(T, p)}$

$$
-\inf _{u \in A} I^{(T, p)}(u) \leq \underline{\lim }_{\epsilon \rightarrow 0} \epsilon \log \mathbb{P}\left(u_{\epsilon} \in A\right) \leq \varlimsup_{\epsilon \rightarrow 0} \epsilon \log \mathbb{P}\left(u_{\epsilon} \in A\right) \leq-\inf _{u \in \bar{A}} I^{(T, p)}(u) .
$$

Indeed, if $u_{\epsilon}$ belongs to $A$, there exists a constant $R$ such that $\left\|u_{\epsilon}\right\|_{X^{(T, p)}} \leq R$. Denoting by $u_{\epsilon}^{R}$ the solution of the following fixed point problem

$$
u_{\epsilon}^{R}(t)=S(t) u_{0}-i \lambda \int_{0}^{t} S(t-s)\left(\left|\left(u_{\epsilon}^{R}-i \sqrt{\epsilon} Z\right)(s)\right|^{2 \sigma}\left(u_{\epsilon}^{R}-i \sqrt{\epsilon} Z\right)(s)\right) \mathbb{1}_{\left\|u_{\epsilon}^{R}\right\|_{\left.X^{(}, p\right)} \leq R} \mathrm{~d} s,
$$

the arguments used previously allow to show that $\sqrt{\epsilon} Z \rightarrow u_{\epsilon}^{R}$ is a continuous mapping from every $X^{\left(T, p^{\prime}\right)}$ into $X^{(T, p)}$ for $p^{\prime}>p$. The result (3.1) with $u_{\epsilon}^{R}$ follows from Varadhan's contraction principle replacing $\mathbf{S}(h)$ by $\mathbf{S}^{R}(h)$ with the truncation in front of the nonlinearity. Finally, the statement follows from the fact that $\left\|u_{\epsilon}\right\|_{X^{(T, p)}} \leq R$ implies that $u_{\epsilon}^{R}=u_{\epsilon}$ and that

$$
\inf _{h \in \mathrm{L}^{2}\left(0, T ; \mathrm{L}^{2}\left(\mathbb{R}^{d}\right)\right): \mathbf{S}^{R}(h) \in \bar{A}}\left\{\|h\|_{\mathrm{L}^{2}\left(0, T ; \mathrm{L}^{2}\left(\mathbb{R}^{d}\right)\right)}^{2}\right\}=\inf _{h \in \mathrm{L}^{2}\left(0, T ; \mathrm{L}^{2}\left(\mathbb{R}^{d}\right)\right): \mathbf{S}(h) \in \bar{A}}\left\{\|h\|_{\mathrm{L}^{2}\left(0, T ; \mathrm{L}^{2}\left(\mathbb{R}^{d}\right)\right)}^{2}\right\} .
$$

Note that writing $\frac{\partial}{\partial t} h$ instead of $h$ in the optimal control problem leads to a rate function consisting in the minimisation of $\frac{1}{2}\|h\|_{H_{0}^{1}\left(0,+\infty ; \mathrm{L}^{2}\left(\mathbb{R}^{d}\right)\right)}^{2}$. This space is somehow the equivalent of the Cameron-Martin space for the Brownian motion. Specifying only the law $\mu$ of $W(1)$ on $\mathrm{H}^{1}\left(\mathbb{R}^{d}\right)$ and dropping $\Phi$ in the control problem would lead to a rate function consisting in the minimisation of $\frac{1}{2}\|h\|_{H_{0}^{1}\left(0,+\infty ; H_{\mu}\right)}^{2}$, where $H_{\mu}$ stands for the RKHS of $\mu$.

The formalism of a LDP stated in the intersection space with a projective limit topology allows, for example, to deduce by contraction, when there is no blow-up in finite time, a variety of sample path LDP on every $X^{(T, p)}$. The rate function could be interpreted as the minimal energy to implement control.

LDP for the family of laws of $u_{\epsilon}(T)$, for a fixed $T$, could be deduced by contraction in the cases of global existence. The rate function is then the minimal energy needed to transfer $u_{0}$ to $x$ from 0 to $T$. An application of this type will be given in Section 6 .

Next section gives a characterization of the support of the law of the solution in our setting. Section 5 is devoted to some consequences of these results on the blow-up time. Finally, in Section 6, applications in nonlinear optics are given.

\section{ThE SUPPORT OF THE LAW OF THE SOLUTION}

Theorem 4.1 (the support theorem). The support of the law of the solution is characterized by

$$
\text { supp } \mu^{u}=\overline{\operatorname{im}} \mathbf{S}^{\mathcal{E}_{\infty}}
$$


and in the cases of global existence by

$$
\operatorname{supp} \mu^{u}=\overline{\operatorname{im~S}}^{\mathcal{X}_{\infty}}
$$

Proof.

Step 1. From Proposition 2.3, given $(T, p)$ in $\mathbb{R}_{+}^{*} \times\left[2, \frac{2}{d-2}\right)$ when $d>2$ or $\mathbb{R}_{+}^{*} \times[2,+\infty)$ when $d=2$ or $d=1$, $\mu^{Z ;(T, p)}$ is a Gaussian measure on a Banach space and its RKHS is im $\mathcal{L}$. Consequently, see [2] Theorem (IX,2;1), its support is $\overline{\mathrm{im} \mathcal{L}}^{X^{(T, p)}}$. Also, from the definition of the image measure we have that

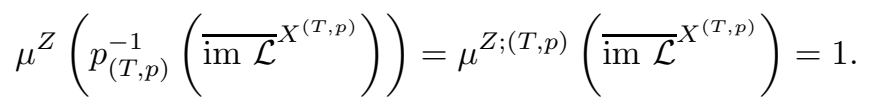

As a consequence the first inclusion follows

$$
\operatorname{supp} \mu^{Z} \subset \bigcap_{(T, p)} p_{(T, p)}^{-1}\left({\overline{\operatorname{im} \mathcal{L}^{X}}}^{X^{(T, p)}}\right)=\overline{\operatorname{im} \mathcal{L}}^{\mathcal{X}_{\infty}}
$$

It then suffices to show that $\operatorname{im} \mathcal{L} \subset \operatorname{supp} \mu^{Z}$. Suppose that $x \notin \operatorname{supp} \mu^{Z}$, then there exists a neighborhood $V$ of $x$ in $\mathcal{X}_{\infty}$, satisfying $V=\bigcap_{i=1}^{n} V^{\left(T_{i}, p_{i}\right)}$ where $V^{\left(T_{i}, p_{i}\right)}$ is a neighborhood of $x$ in $X^{\left(T_{i}, p_{i}\right)}, n$ is a finite integer and $\left(T_{i}, p_{i}\right)$ a finite sequence of elements of $\mathbb{R}_{+}^{*} \times\left[2, \frac{2}{d-2}\right)$ when $d>2$ or $\mathbb{R}_{+}^{*} \times[2,+\infty)$ when $d=2$ or $d=1$, such that $\mu^{Z}(V)=0$. It can be shown that $\bigcap_{i=1}^{n} X^{\left(T_{i}, p_{i}\right)}$ is still a separable Banach space. It is such that $\mathcal{X}_{\infty}$ is continuously embedded into it, and such that the Borel direct image probability measure is a Gaussian measure of RKHS im $\mathcal{L}$. The support of this measure is then the closure of im $\mathcal{L}$ for the topology defined by the maximum of the norms on each factor. Thus, $V \cap$ im $\mathcal{L}=\emptyset$ and $x \notin \operatorname{im} \mathcal{L}$.

Step 2. We conclude using the continuity of $\mathcal{G}$.

Indeed since $\mathcal{G}(\operatorname{im} \mathcal{L}) \subset \overline{\mathcal{G}(\operatorname{im~} \mathcal{L})}^{\mathcal{E}}, \operatorname{im} \mathcal{L} \subset \mathcal{G}^{-1}\left(\overline{\mathcal{G}(\operatorname{im~} \mathcal{L})}^{\mathcal{E}_{\infty}}\right)$. Since $\mathcal{G}$ is continuous, the right side is a closed set of $\mathcal{X}_{\infty}$ and from step 1 ,

$$
\operatorname{supp} \mu^{Z} \subset \mathcal{G}^{-1}\left(\overline{\operatorname{im}(\mathcal{G} \circ \mathcal{L})}^{\mathcal{E}_{\infty}}\right)
$$

and

$$
\mu^{Z}\left(\mathcal{G}^{-1}\left(\overline{\operatorname{im~S}}^{\mathcal{E}_{\infty}}\right)\right)=1
$$

thus

$$
\operatorname{supp} \mu^{u} \subset \overline{\operatorname{im~S}}^{\mathcal{E}_{\infty}} \text {. }
$$

Suppose that $x \notin \operatorname{supp} \mu^{u}$, there exists a neighborhood $V$ of $x$ in $\mathcal{E}_{\infty}$ such that $\mu^{u}(V)=\mu^{Z}\left(\mathcal{G}^{-1}(V)\right)=0$, consequently $\mathcal{G}^{-1}(V) \bigcap \operatorname{im} \mathcal{L}=\emptyset$ and $x \notin \operatorname{im} \mathbf{S}$. This gives reverse inclusion.

The same arguments hold replacing $\mathcal{E}_{\infty}$ by $\mathcal{X}_{\infty}$.

Note that the result of step 2 is general and gives that the support of the direct images $\mu^{E}$ of the law $\mu^{u}$ by any continuous mapping $f$ from either $\mathcal{E}_{\infty}$ or $\mathcal{X}_{\infty}$ into a topological vector space $E$ is $\overline{\operatorname{im}(f \circ \mathbf{S})}^{E}$. For example, in the cases of global existence, given a positive $T$, the support of the law in $\mathrm{H}^{1}\left(\mathbb{R}^{d}\right)$ of $u(T)$ is $\overline{\operatorname{im} \mathbf{S}(T)} \mathrm{H}^{1}\left(\mathbb{R}^{d}\right)$.

Remark 4.2. Remark that the LDP and support theorem may be proved for more general driving noises provided that the stochastic convolution remains a Gaussian process. The case of a noise derived from a fractional Wiener process which is a one parameter generalization of the usual Wiener process has been studied. The results will appear elsewhere. 


\section{Applichtions to the Blow-up time}

In this section the equation with a focusing nonlinearity, i.e. $\lambda=1$, is considered. In this case, it is known that some solutions of the deterministic equation blow up in finite time for a critical or subcritical nonlinearity. It has been proved in Section 2.2 that $\mathcal{T}$ is a measurable mapping from $\mathcal{E}_{\infty}$ to $[0,+\infty]$, both spaces are equipped with their Borel $\sigma$-fields. Incidentally, $\mathcal{T}(u)$ is a $\mathcal{F}_{t}$-stopping time. Also, if $B$ is a Borel set of $[0,+\infty], \mathbb{P}(\mathcal{T}(u) \in B)=\mu^{u}\left(\mathcal{T}^{-1}(B)\right)$.

The support theorem allows us to determine whether an open or a closed set of the form $\mathcal{T}^{-1}(B)$ is such that $\mu^{u}\left(\mathcal{T}^{-1}(B)\right)>0$ or $\mu^{u}\left(\mathcal{T}^{-1}(B)\right)<1$ respectively. An application of this fact is given in Proposition 5.1 . For a Borel set $B$ such that $\left\{\operatorname{Int}\left(\mathcal{T}^{-1}(B)\right)\right\} \cap \overline{\mathrm{im} \mathrm{S}}^{\mathcal{E}_{\infty}}$ is nonempty, where $\operatorname{Int}\left(\mathcal{T}^{-1}(B)\right)$ stands for the interior set of $\mathcal{T}^{-1}(B), \mathbb{P}(\mathcal{T}(u) \in B)>0$ holds.

Also, $\mathcal{T}$ is not continuous and Varadhan's contraction principle does not allow to obtain a LDP for the law of the blow-up time. Nonetheless, the LDP for the family $\left(\mu^{u_{\epsilon}}\right)_{\epsilon>0}$ gives the interesting result that

$$
-\inf _{u \in \operatorname{Int}\left(\mathcal{T}^{-1}(B)\right)} I(u) \leq \underline{\lim }_{\epsilon \rightarrow 0} \epsilon \log \mathbb{P}\left(\mathcal{T}\left(u_{\epsilon}\right) \in B\right) \leq \varlimsup_{\epsilon \rightarrow 0} \epsilon \log \mathbb{P}\left(\mathcal{T}\left(u_{\epsilon}\right) \in B\right) \leq-\inf _{u \in \mathcal{T}^{-1}(B)} I(u) .
$$

Note also that the interior or the closure of sets in $\mathcal{E}_{\infty}$ are difficult to characterize. In that respect, the semicontinuity of $\mathcal{T}$ makes the sets $(T,+\infty]$ and $[0, T]$ particularly interesting.

\subsection{Probability of blow-up after time $\mathbf{T}$}

Proposition 5.1. If $u_{0} \in \mathrm{H}^{3}\left(\mathbb{R}^{d}\right)$ and the range of $\Phi$ is dense then for every positive $T$,

$$
\mathbb{P}(\mathcal{T}(u)>T)>0
$$

Proof. Since $\mathcal{T}$ is lower semicontinuous, $\mathcal{T}^{-1}((T,+\infty])$ is an open set.

Consider $H=-\Delta u_{0}-\left|u_{0}\right|^{2 \sigma} u_{0}$ which satisfies $\mathcal{G} \circ \Lambda(H)=u_{0}$, where $\Lambda$ has been defined in Section 2.1, then $\mathcal{T}(\mathbf{S}(H))=+\infty$. Also, using $\Phi$ one defines, in a natural way, an operator from $\mathrm{L}_{\text {loc }}^{2}\left(0,+\infty ; \mathrm{L}^{2}\left(\mathbb{R}^{d}\right)\right)$ into $\mathrm{L}_{l o c}^{2}\left(0,+\infty ; \mathrm{H}^{1}\left(\mathbb{R}^{d}\right)\right)$ and it can be shown, that it still has a dense range. Consequently, there exists a sequence $\left(h_{n}\right)_{n \in \mathbb{N}}$ of $\mathrm{L}_{l o c}^{2}\left(0,+\infty ; \mathrm{L}^{2}\left(\mathbb{R}^{d}\right)\right)$ functions such that $\left(\Phi\left(h_{n}\right)\right)_{n \in \mathbb{N}}$ converges to $H$ in $\mathrm{L}_{l o c}^{2}\left(0,+\infty ; \mathrm{H}^{1}\left(\mathbb{R}^{d}\right)\right)$.

Using the semicontinuity of $\mathcal{T}$, the continuity of $\mathcal{G}$, the fact that $\mathbf{S}=\mathcal{G} \circ \Lambda \circ \Phi$, the following lemma and the fact that $\mathrm{L}_{l o c}^{2}\left(0,+\infty ; \mathrm{H}^{1}\left(\mathbb{R}^{d}\right)\right)$ is continuously embedded in $\mathrm{L}_{l o c}^{1}\left(0,+\infty ; \mathrm{H}^{1}\left(\mathbb{R}^{d}\right)\right), \underline{\lim }_{n \rightarrow \infty} \mathcal{T}\left(\mathbf{S}\left(h_{n}\right)\right) \geq+\infty$, i.e. $\lim _{n \rightarrow \infty} \mathcal{T}\left(\mathbf{S}\left(h_{n}\right)\right)=+\infty$, follows. Therefore $\mathcal{T}\left(\mathbf{S}\left(h_{n}\right)\right)>T$ for $n$ large enough and $\mathcal{T}^{-1}((T,+\infty]) \cap(\mathrm{im} \mathbf{S})$ is nonempty.

The conclusion follows then from the support theorem.

As a corollary, taking the complement of $\mathcal{T}^{-1}((T,+\infty]), \mathbb{P}(\mathcal{T}(u) \leq T)<1$ follows. This is related to the results of [8] where it is proved that for every positive $T, \mathbb{P}(\mathcal{T}(u)<T)>0$ and to the graphs in Section 4 of $[10]$.

Lemma 5.2. The operator $\Lambda$, defined in Section 2.1, is continuous from $\mathrm{L}_{\text {loc }}^{1}\left(0,+\infty ; \mathrm{H}^{1}\left(\mathbb{R}^{d}\right)\right)$ into $\mathcal{X}_{\infty}$.

Proof. The result follows from $i i /$ of the Strichartz estimates when $s=1$ and $\rho=2$, the fact that the partial derivatives with respect to one space variable commutes with both the integral and the group and the definition of the projective limit topology.

The following result holds when the intensity of the noise converges to zero.

Proposition 5.3. If $u_{0} \in \mathrm{H}^{3}\left(\mathbb{R}^{d}\right)$, the range of $\Phi$ is dense and $T \geq \mathcal{T}\left(u_{d}\right)$, where $u_{d}$ is the solution of the deterministic NLS equation with initial datum $u_{0}$, there exists $c$ in $[0,+\infty)$ such that

$$
\underline{\lim }_{\epsilon \rightarrow 0} \epsilon \log \mathbb{P}\left(\mathcal{T}\left(u_{\epsilon}\right)>T\right) \geq-c
$$


Proof. Define

$$
L^{(T,+\infty]}=\frac{1}{2} \inf _{h \in \mathrm{L}^{2}\left(0,+\infty ; \mathrm{L}^{2}\left(\mathbb{R}^{d}\right)\right): \mathcal{T}(\mathbf{S}(h))>T}\left\{\|h\|_{\mathrm{L}^{2}\left(0,+\infty ; \mathrm{L}^{2}\left(\mathbb{R}^{d}\right)\right)}^{2}\right\}
$$

The result follows then from

$$
-L^{(T,+\infty]} \leq \underline{\lim }_{\epsilon \rightarrow 0} \epsilon \log \mathbb{P}\left(\mathcal{T}\left(u_{\epsilon}\right)>T\right)
$$

and that, from the arguments of the proof of Proposition 5.1, for every $T$ such that $T \geq \mathcal{T}\left(u_{d}\right)$ the set $\left\{h \in \mathrm{L}^{2}\left(0,+\infty ; \mathrm{L}^{2}\left(\mathbb{R}^{d}\right)\right): \mathcal{T}(\mathbf{S}(h))>T\right\}$ is nonempty.

Remark 5.4. The assumption that $u_{0} \in \mathrm{H}^{3}\left(\mathbb{R}^{d}\right)$ could be dropped using similar arguments as in Proposition 3.3 of $[8]$.

Note that the LDP does not give interesting information on the upper bound even if the bounds have been sharpened using the rather strong projective limit topology. It is zero since $h=0$ belongs to $\overline{\mathcal{T}-1((T,+\infty])}$ as for every $T>0, \overline{\mathcal{T}}-1((T,+\infty])=\mathcal{E}_{\infty}$. Indeed, if a function $f$ of $\mathcal{E}_{\infty}$ is given and blows up at a particular time $\mathcal{T}(f)$ such that $T>\mathcal{T}(f)$, it is possible to build a sequence $\left(f_{n}\right)_{n \in \mathbb{N}}$ of functions of $\mathcal{E}_{\infty}$ equal to $f$ on $\left[0, \mathcal{T}(f)-\frac{1}{n}\right]$ and such that $\mathcal{T}\left(f_{n}\right)>T$. The same problem will appear in the next section where the LDP gives a lower bound equal to $-\infty$. Indeed, $\operatorname{Int}\left(\mathcal{T}^{-1}([0, T])\right)$ is the complement of the above and thus an empty set. To overcome this problem the approximate blow-up time is introduced. Note also that it is possible that $L^{(T,+\infty]}=0$.

Also, the case $T<\mathcal{T}\left(u_{d}\right)$ has not been treated. Indeed, the associated event is not a large deviation event and the LDP only gives that $\lim _{\epsilon \rightarrow 0} \epsilon \log \mathbb{P}\left(\mathcal{T}\left(u_{\epsilon}\right)>T\right)=0$.

\subsection{Probability of blow-up before time $\mathbf{T}$}

In that case we obtain

$$
-\infty \leq \underline{\lim }_{\epsilon \rightarrow 0} \epsilon \log \mathbb{P}\left(\mathcal{T}\left(u_{\epsilon}\right) \leq T\right) \leq \varlimsup_{\epsilon \rightarrow 0} \epsilon \log \mathbb{P}\left(\mathcal{T}\left(u_{\epsilon}\right) \leq T\right) \leq-U^{[0, T]}
$$

where $U^{[0, T]}=\frac{1}{2} \inf _{h \in \mathrm{L}^{2}\left(0,+\infty ; \mathrm{L}^{2}\left(\mathbb{R}^{d}\right)\right): \mathcal{T}(\mathbf{S}(h)) \leq T}\left\{\|h\|_{\mathrm{L}^{2}\left(0,+\infty ; \mathrm{L}^{2}\left(\mathbb{R}^{d}\right)\right)}^{2}\right\}$.

Proposition 5.5. If $T<\mathcal{T}\left(u_{d}\right)$,

$$
\varlimsup_{\epsilon \rightarrow 0} \epsilon \log \mathbb{P}\left(\mathcal{T}\left(u_{\epsilon}\right) \leq T\right) \leq-U^{[0, T]}<0
$$

Proof. Let $\left(h_{n}\right)_{n \in \mathbb{N}}$ be a sequence of $\mathrm{L}^{2}\left(0,+\infty ; \mathrm{L}^{2}\left(\mathbb{R}^{d}\right)\right)$ functions converging to zero. It follows from Lemma 5.2 and the fact that $\mathrm{L}^{2}\left(0,+\infty ; \mathrm{H}^{1}\left(\mathbb{R}^{d}\right)\right)$ is continuously embedded into $\mathrm{L}_{\text {loc }}^{1}\left(0,+\infty ; \mathrm{H}^{1}\left(\mathbb{R}^{d}\right)\right)$ that $\mathbf{S}=\mathcal{G} \circ \Lambda \circ \Phi$ is continuous from $\mathrm{L}^{2}\left(0,+\infty ; \mathrm{L}^{2}\left(\mathbb{R}^{d}\right)\right)$ into $\mathcal{E}_{\infty}$. Also, from the semicontinuity of $\mathcal{T}, \underline{\lim }_{n \rightarrow+\infty} \mathcal{T}\left(\mathbf{S}\left(h_{n}\right)\right) \geq \mathcal{T}\left(u_{d}\right)$. Thus there exists $N$ large enough such that for every $n \geq N, \mathcal{T}\left(\mathbf{S}\left(h_{n}\right)\right)>T$. As a consequence we obtain that necessarily $U^{[0, T]}>0$.

When $T \geq \mathcal{T}\left(u_{d}\right)$, the probability is not supposed to tend to zero. Also, as $h=0$ is a solution, the upper bound is zero and none of the bounds are interesting.

\subsection{Bounds for the approximate blow-up time}

To overcome the limitation that $\overline{\mathcal{T}^{-1}((T,+\infty])}=\mathcal{E}_{\infty}$, which does not allow to have two interesting bounds simultaneously, we introduce for every positive $R$ the mappings $\mathcal{T}_{R}$ defined for $f \in \mathcal{E}_{\infty}$ by

$$
\mathcal{T}_{R}(f)=\inf \left\{t \in[0,+\infty):\|f(t)\|_{\mathrm{H}^{1}\left(\mathbb{R}^{d}\right)} \geq R\right\}
$$

It corresponds to the approximation of the blow-up time used in [10]. We obtain the following bounds. 
Proposition 5.6. When $T \geq \mathcal{T}_{R}\left(u_{d}\right)$, the following inequality holds

$$
-c<-L_{R}^{(T,+\infty]} \leq \underline{\lim }_{\epsilon \rightarrow 0} \epsilon \log \mathbb{P}\left(\mathcal{T}_{R}\left(u_{\epsilon}\right)>T\right) \leq \varlimsup_{\epsilon \rightarrow 0} \epsilon \log \mathbb{P}\left(\mathcal{T}_{R}\left(u_{\epsilon}\right)>T\right) \leq-\sup _{\alpha>0} L_{R+\alpha}^{(T,+\infty]}
$$

Also, when $T<\mathcal{T}_{R}\left(u_{d}\right)$, we have that

$$
-\inf _{\alpha>0} U_{R+\alpha}^{[0, T]} \leq \underline{\lim }_{\epsilon \rightarrow 0} \epsilon \log \mathbb{P}\left(\mathcal{T}_{R}\left(u_{\epsilon}\right) \leq T\right) \leq \varlimsup_{\epsilon \rightarrow 0} \epsilon \log \mathbb{P}\left(\mathcal{T}_{R}\left(u_{\epsilon}\right) \leq T\right) \leq-U_{R}^{[0, T]}<0
$$

In the above $c$ is nonnegative and the numbers $L_{R}^{(T,+\infty]}$ and $U_{R}^{[0, T]}$ are defined as $L^{(T,+\infty]}$ and $U^{[0, T]}$ replacing $\mathcal{T}$ by $\mathcal{T}_{R}$.

Proof. The result follows from the facts that $\mathcal{T}_{R}$, which is not continuous, is lower semicontinuous, that for every $\alpha>0, \overline{\mathcal{T}_{R}^{-1}((T,+\infty])} \subset \mathcal{T}_{R+\alpha}^{-1}((T,+\infty])$, thus $\mathcal{T}_{R+\alpha}^{-1}([0, T]) \subset \operatorname{Int}\left(\mathcal{T}_{R}^{-1}([0, T])\right)$ and from the arguments used in the proofs of the last two propositions.

We also obtain the following estimates of other large deviation events.

Corollary 5.7. If $S, T<\mathcal{T}_{R}\left(u_{d}\right)$, for every $c>0$, there exists $\epsilon_{0}>0$ such that if $\epsilon \leq \epsilon_{0}$,

$$
\exp \left(-\frac{\inf _{\alpha>0} U_{R+\alpha}^{[0, T]}+c}{\epsilon}\right)\left(1-\exp \left(-\frac{U_{R}^{[0, S]}-\inf _{\alpha>0} U_{R+\alpha}^{[0, T]}}{\epsilon}\right)\right) \leq \mathbb{P}\left(S<\mathcal{T}_{R}\left(u_{\epsilon}\right) \leq T\right)
$$

and

$$
\mathbb{P}\left(S<\mathcal{T}_{R}\left(u_{\epsilon}\right) \leq T\right) \leq \exp \left(-\frac{U_{R}^{[0, T]}-c}{\epsilon}\right)\left(1-\exp \left(-\frac{\inf _{\alpha>0} U_{R+\alpha}^{[0, S]}-U_{R}^{[0, T]}}{\epsilon}\right)\right) .
$$

If $S, T>\mathcal{T}_{R}\left(u_{d}\right)$, for every positive $c$, there exists a positive $\epsilon_{0}$ such that if $\epsilon \leq \epsilon_{0}$,

$$
\exp \left(-\frac{L_{R}^{(S,+\infty]}+c}{\epsilon}\right)\left(1-\exp \left(-\frac{\sup _{\alpha>0} L_{R+\alpha}^{(T,+\infty]}-L_{R}^{(T,+\infty]}}{\epsilon}\right)\right) \leq \mathbb{P}\left(S<\mathcal{T}_{R}\left(u_{\epsilon}\right) \leq T\right)
$$

and

$$
\mathbb{P}\left(S<\mathcal{T}_{R}\left(u_{\epsilon}\right) \leq T\right) \leq \exp \left(-\frac{\sup _{\alpha>0} L_{R+\alpha}^{(S,+\infty]}-c}{\epsilon}\right)\left(1-\exp \left(-\frac{L_{R}^{(T,+\infty]}-\sup _{\alpha>0} L_{R+\alpha}^{(S,+\infty]}}{\epsilon}\right)\right)
$$

Proof. When $S, T<\mathcal{T}_{R}\left(u_{d}\right)$, the result follows from the inequalities and from the fact that

$$
\mathbb{P}\left(S<\mathcal{T}_{R}\left(u_{\epsilon}\right) \leq T\right)=\mathbb{P}\left(\left\{\mathcal{T}_{R}\left(u_{\epsilon}\right) \leq T\right\} \backslash\left\{\mathcal{T}_{R}\left(u_{\epsilon}\right) \leq S\right\}\right)=\mathbb{P}\left(\mathcal{T}_{R}\left(u_{\epsilon}\right) \leq T\right)\left(1-\frac{\mathbb{P}\left(\mathcal{T}_{R}\left(u_{\epsilon}\right) \leq S\right)}{\mathbb{P}\left(\mathcal{T}_{R}\left(u_{\epsilon}\right) \leq T\right)}\right)
$$

When $S, T>\mathcal{T}_{R}\left(u_{d}\right)$, we use

$$
\mathbb{P}\left(S<\mathcal{T}_{R}\left(u_{\epsilon}\right) \leq T\right)=\mathbb{P}\left(\left\{\mathcal{T}_{R}\left(u_{\epsilon}\right)>S\right\} \backslash\left\{\mathcal{T}_{R}\left(u_{\epsilon}\right)>T\right\}\right)=\mathbb{P}\left(\mathcal{T}_{R}\left(u_{\epsilon}\right)>S\right)\left(1-\frac{\mathbb{P}\left(\mathcal{T}_{R}\left(u_{\epsilon}\right)>T\right)}{\mathbb{P}\left(\mathcal{T}_{R}\left(u_{\epsilon}\right)>S\right)}\right)
$$




\section{Applications to nonlinear optics}

The NLS equation when $d=\lambda=\sigma=1$ is called the noisy cubic focusing nonlinear Schrödinger equation. It is a model used in nonlinear optics. Recall that for the above values of the parameters the solutions are global. The variable $t$ stands for the one dimensional space coordinate and $x$ for the time. The deterministic equation is such that there exists a particular class of solutions, which are localized in space (here time), that propagate at a finite constant velocity and keep the same shape. These solutions are called solitons or solitary waves. The functions

$$
\Psi_{\eta}(t, x)=\sqrt{2} \eta \exp \left(-i \eta^{2} t\right) \operatorname{sech}(\eta x), \eta>0
$$

form a family of solitons. They are used in optical fibers as information carriers to transmit the datum 0 or 1 at high bit rates over long distances. The noise stands for the noise produced by in-line amplifiers.

Let $u_{\epsilon}$ denotes the solution with $u_{0}(\cdot)=\Psi_{1}(0, \cdot)$ as initial datum and $\epsilon$ as noise intensity like in Section 3 and $u_{\epsilon}^{n}$ denotes the solution with null initial datum and the same noise intensity. The mass of $u_{0}$ is 4 .

At a particular coordinate $T$ of the fiber, when a window $[-l, l]$ is given, the square of the $\mathrm{L}^{2}(-l, l)-$ norm, or measured mass, is recorded. It is close to the mass in the deterministic case for sufficiently high $l$ since the wave is localized. A decision criterium is to accept that we have 1 if the measured mass is above a certain threshold and 0 otherwise. We set a threshold of the form $4(1-\gamma)$, where $\gamma$ is a real number in $[0,1]$.

As the soliton is progressively distorted by the noise, it is possible either to wrongly decide that the source has emitted a 1, or to wrongly discard a 1. The two error probabilities consist of

$$
\mathbb{P}_{\epsilon}^{\mid 0}=\mathbb{P}\left(\int_{-l}^{l}\left|u_{\epsilon}^{n}(T, x)\right|^{2} \mathrm{~d} x \geq 4(1-\gamma)\right)
$$

and

$$
\mathbb{P}_{\epsilon}^{\mid 1}=\mathbb{P}\left(\int_{-l}^{l}\left|u_{\epsilon}(T, x)\right|^{2} \mathrm{~d} x<4(1-\gamma)\right) .
$$

In modern communication systems the error rate is less than $10^{-9}$ which is beyond the scope of statistics, moreover due to the nonlinearity of the system the measured mass does not have a gaussian law. This justifies that we use theoretical arguments to characterize these error probabilities. We show in this section how the LDP applies to this problem.

We obtain similar results, in the case of an unbounded window, as in reference [16] for the first error probability and as [17] for the second error probability. In these articles the heuristic argument of the collective coordinates is used. This is a physical argument which unables to reduce the problem to a finite dimensional system involving modulated parameters. In [16], the authors explain what the leading parameters are and reduce the problem to a three dimensional problem, that the fluctuations of the parameters are described by SDEs where the noises are some spatial integrals of the initial noise and that the averaging over the initial noise is equivalent to averaging over new noises with zero cross correlations. They explain that a decrease in the soliton power $Q_{\epsilon}=\frac{M\left(u_{\epsilon}(T)\right)^{2}}{4}$ and a timing jitter $T_{\epsilon}=\frac{\int_{-\infty}^{+\infty} x\left|u_{\epsilon}(T, x)\right|^{2} \mathrm{~d} x}{M\left(u_{\epsilon}(T)\right)^{2}}$, which characterizes the shifts in the arrival time of the pulse, are mainly responsible for the loss of the pulse. Thus they write down the probability density function of the joint law of the two processes, using a formalism called the instanton formalism, as a quantity which is the averaging over the noise of the path integral over arbitrary functions for the modulated parameters, taking into account the finite dimensional evolution, of the exponential of an integral over $t$ in $[0, T]$ of the so called effective Lagrangian. Finally they compute the path integral using a saddle-point approximation with boundary conditions and obtain an expression of the probability density function in the small noise asymptotic. Details on the calculation are given in [17]. The overall argument seems very difficult to justify rigorously. In particular, the reduction to a three dimensional problem is obtained by minimizing the Lagrangian on a small space of curves whereas NLS is obtained by minimizing over all paths. Note that they recover analytically the empirical Gordon-Haus effect that the dispersion in timing is much larger than that of the mass. The authors also explain that, for the first error probability, the optimal way to create a large signal is to grow a soliton 
and obtain a small noise asymptotic expression of the probability density function of the amplitude at the coordinate $T$ of the solution with null initial datum.

In the following we make the assumption that im $\Phi$ has a dense range. Indeed, from the arguments used in the proof of Proposition 5.3, it is needed for controllability issues to guarantee that the infima are not taken over empty sets. Also $T$ is fixed, $\gamma_{0} \in\left(0, \frac{1}{2}\right)$ is fixed and the size $l$ of the window is such that

$$
\int_{-l}^{l}\left|u_{d}(T, x)\right|^{2} \mathrm{~d} x \wedge \int_{-l}^{l}\left|\Psi_{1}(0, x)\right|^{2} \mathrm{~d} x>4\left(1-\frac{\gamma_{0}}{2}\right) .
$$

We finally stress that for the events we study in dimension $d=1$ we could consider a $L^{2}(\mathbb{R})$ setting instead of a $H^{1}(\mathbb{R})$ setting. However we chose to work in $\mathrm{H}^{1}(\mathbb{R})$ to keep the coherence of the article. The $\mathrm{H}^{1}(\mathbb{R})$ setting is necessary when we consider higher dimensions or larger powers of nonlinearities and study events like

$$
\left\{\int_{-l}^{l}\left|u_{\epsilon}^{n}(T, x)\right|^{2} \mathrm{~d} x \geq 4-\gamma, \mathcal{T}\left(u_{\epsilon}^{n}\right)>T\right\} \quad \text { or } \quad\left\{\int_{-l}^{l}\left|u_{\epsilon}(T, x)\right|^{2} \mathrm{~d} x<4-\gamma, \mathcal{T}\left(u_{\epsilon}\right)>T\right\} .
$$

The LDP proved herein allows to state the following proposition.

Proposition 6.1. For every $\gamma$ in $\left[\gamma_{0}, 1-\gamma_{0}\right]$ besides an at most countable set of points, the following equivalents for the probabilities of error hold

$$
\begin{aligned}
& \lim _{\epsilon \rightarrow 0} \epsilon \log \mathbb{P}_{\epsilon}^{\mid 0}=-\frac{1}{2} \inf _{h \in \mathrm{L}^{2}\left(0,+\infty ; \mathrm{L}^{2}(\mathbb{R})\right): \int_{-l}^{l}|\tilde{\mathbf{S}}(h)(T, x)|^{2} \mathrm{~d} x \geq 4(1-\gamma)}\left\{\|h\|_{\mathrm{L}^{2}\left(0,+\infty ; \mathrm{L}^{2}(\mathbb{R})\right)}^{2}\right\} \\
& \lim _{\epsilon \rightarrow 0} \epsilon \log \mathbb{P}_{\epsilon}^{\mid 1}=-\frac{1}{2} \inf _{h \in \mathrm{L}^{2}\left(0,+\infty ; \mathrm{L}^{2}(\mathbb{R})\right): \int_{-l}^{l}|\mathbf{S}(h)(T, x)|^{2} \mathrm{~d} x<4(1-\gamma)}\left\{\|h\|_{\mathrm{L}^{2}\left(0,+\infty ; \mathrm{L}^{2}(\mathbb{R})\right)}^{2}\right\}
\end{aligned}
$$

where $\mathbf{S}(h)$ and $\tilde{\mathbf{S}}(h)$ correspond to the usual skeleton with respectively a soliton and a null initial datum. Moreover, both infima are positive numbers.

Proof. The mapping $\varphi$ from $\mathcal{X}_{\infty}$ into $\mathbb{R}^{+}$such that $\varphi(f)=\int_{-l}^{l}|f(T, x)|^{2} \mathrm{~d} x$ is continuous. Therefore, the direct image measures $\left(\varphi_{*} \mu^{u_{\epsilon}}\right)_{\epsilon \geq 0}$ and $\left(\varphi_{*} \mu^{u_{\epsilon}^{n}}\right)_{\epsilon \geq 0}$ satisfy LDP of speed $\epsilon$ and good rate functions respectively

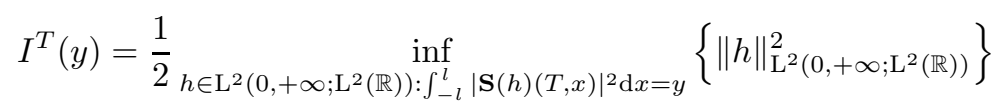

and $J^{T}$ where $\mathbf{S}$ is replaced by $\tilde{\mathbf{S}}$. Consequently,

$$
\forall i \in\{0,1\},-L^{i}(\gamma) \leq \underline{\lim }_{\epsilon \rightarrow 0} \epsilon \log \mathbb{P}_{\epsilon}^{i} \leq \varlimsup_{\epsilon \rightarrow 0} \epsilon \log \mathbb{P}_{\epsilon}^{\mid i} \leq-U^{i}(\gamma)
$$

where

$$
\begin{array}{ll}
L^{0}(\gamma)=\inf _{y \in(4(1-\gamma),+\infty)} J^{T}(y), \quad U^{0}(\gamma)=\inf _{y \in[4(1-\gamma),+\infty)} J^{T}(y), \\
L^{1}(\gamma)=\inf _{y \in[0,4(1-\gamma))} I^{T}(y), & U^{1}(\gamma)=\inf _{y \in[0,4(1-\gamma)]} I^{T}(y) .
\end{array}
$$

For every $\delta>0, U^{0}(\gamma) \leq L^{0}(\gamma) \leq U^{0}(\gamma-\delta)$ and $U^{1}(\gamma) \leq L^{1}(\gamma) \leq U^{1}(\gamma+\delta)$ hold

The function $\gamma \mapsto U^{0}(\gamma)$ is positive and decreasing. Also, since the range of $\Phi$ is dense, there exists a sequence $\left(h_{n}^{0}\right)_{n \in \mathbb{N}}$ of functions of $\mathrm{L}^{2}\left(0,+\infty ; \mathrm{L}^{2}(\mathbb{R})\right)$ so that $\Phi h_{n}$ converges to

$$
H^{0}(t)=i \frac{\mathrm{d}}{\mathrm{d} t} u^{0}-\Delta u^{0}-\lambda\left|u^{0}\right|^{2 \sigma} u^{0}
$$


where

$$
u^{0}(t)=\mathbb{1}_{t \leq T} \frac{t}{T} \Psi_{1}(0, \cdot)
$$

and by the continuity proved in Section $5.1\left(\varphi \circ \tilde{\mathbf{S}}\left(h_{n}^{0}\right)\right)_{n \in \mathbb{N}}$ converges to $\varphi \circ \tilde{\mathbf{S}}\left(H^{0}\right)>4\left(1-\frac{\gamma_{0}}{2}\right)>4\left(1-\gamma_{0}\right)$. Consequently, $h_{n}^{0}$ belongs to the minimizing set for $n$ large enough. Thus, $U^{0}\left(\gamma_{0}\right)<+\infty$ follows. Consequently, the function $\gamma \mapsto U^{0}(\gamma)$ possesses an at most countable set of points of discontinuity.

Similarly, the function $\gamma \mapsto U^{1}(\gamma)$ is a bounded increasing function. Also, if $\left(h_{n}^{1}\right)_{n \in \mathbb{N}}$ and $H^{1}(t)$ are defined as previously replacing $u^{0}(t)$ by

$$
u^{1}(t)=\mathbb{1}_{t \leq T}\left(1-\left(1-\sqrt{\frac{\gamma_{0}}{2}}\right) \frac{t}{T}\right) \Psi_{1}(0, \cdot)
$$

the sequence $\left(\varphi \circ \mathbf{S}\left(h_{n}^{1}\right)\right)_{n \in \mathbb{N}}$ converges to $\varphi \circ \mathbf{S}\left(H^{1}\right) \leq 2 \gamma_{0}=4\left(1-\left(1-\frac{\gamma_{0}}{2}\right)\right)$. Thus, for $n$ large enough $h_{n}^{1}$ belongs to the minimizing set. Consequently, the function $\gamma \mapsto U^{1}(\gamma)$ has an at most countable set of points of discontinuity. Thus, for a well chosen $\gamma$, letting $\delta$ converge to zero, we obtain for $i \in\{0,1\}$ that $L^{i}(\gamma)=U^{i}(\gamma)$ and the equivalents follow.

From the arguments used in the proof of Proposition 5.5, $\tilde{\mathbf{S}}$ is a continuous mapping from $\mathrm{L}^{2}\left(0,+\infty ; \mathrm{H}^{1}(\mathbb{R})\right)$ into $\mathcal{X}_{\infty}$. Since $\varphi$ is continuous, if $\left(H_{n}\right)_{n \in \mathbb{N}}$ is a sequence of functions converging to zero in $\mathrm{L}^{2}\left(0,+\infty ; \mathrm{H}^{1}(\mathbb{R})\right)$ then $\left(\varphi \circ \tilde{\mathbf{S}}\left(H_{n}\right)\right)_{n \in \mathbb{N}}$ converges to $\varphi \circ \tilde{\mathbf{S}}(0)=0$. Similarly we obtain that $\left(\varphi \circ \mathbf{S}\left(H_{n}\right)\right)_{n \in \mathbb{N}}$ converges to $\varphi \circ \mathbf{S}(0)>$ $4\left(1-\frac{\gamma_{0}}{2}\right)$. We have now proved the last point of our result that is both infima are positive.

In the two following sections we concentrate on the mass, we take $l=+\infty$ as if the window were not bounded. Somehow, if we forget the coefficient, we concentrate on the tails of the marginal law of the soliton power when the initial datum is a soliton or the tails of the amplitude of the solution with null initial datum as $\epsilon$ converges to zero. We recall, it has been pointed out in the introduction, that the mass is no longer preserved in the stochastic case and is such that its expected value increases.

\subsection{Upper bounds}

The norm of the linear continuous operator $\Phi$ of $\mathrm{L}^{2}(\mathbb{R})$ is thereafter denoted by $\|\Phi\|_{c}$.

Proposition 6.2. For every positive $T, \gamma$ in $[0,1]$, and every operator $\Phi$ in $\mathcal{L}_{2}\left(\mathrm{~L}^{2}(\mathbb{R}), \mathrm{H}^{1}(\mathbb{R})\right)$, the inequalities

$$
\varlimsup_{\epsilon \rightarrow 0} \epsilon \log \mathbb{P}_{\epsilon}^{\mid 0} \leq-\frac{1-\gamma}{2 T\|\Phi\|_{c}^{2}}
$$

and

$$
\varlimsup_{\epsilon \rightarrow 0} \epsilon \log \mathbb{P}_{\epsilon}^{\mid 1} \leq-\frac{\gamma^{2}}{2 T\|\Phi\|_{c}^{2}(1+\gamma)^{2}}
$$

hold.

Proof. Multiplying by $-i \bar{u}$ the equation

$$
i \frac{\mathrm{d}}{\mathrm{d} t} u-\Delta u-\lambda|u|^{2 \sigma} u=\Phi h,
$$

integrating over time and space and taking the real part gives that

$$
\|u(T)\|_{\mathrm{L}^{2}(\mathbb{R})}^{2}-\left\|u_{0}\right\|_{\mathrm{L}^{2}(\mathbb{R})}^{2}=2 \Re\left(-i \int_{0}^{T} \int_{\mathbb{R}} \Phi h \bar{u} \mathrm{~d} x \mathrm{~d} t\right) .
$$


First bound: The boundary conditions $\|u(T)\|_{L^{2}(\mathbb{R})}^{2} \geq 4(1-\gamma)$ and $u_{0}=0$ along with Cauchy Schwarz inequality imply both that

and that

$$
4(1-\gamma) \leq 2\|\Phi\|_{c}\|h\|_{\mathrm{L}^{2}\left(0, T ; \mathrm{L}^{2}(\mathbb{R})\right)}\|u\|_{\mathrm{L}^{2}\left(0, T ; \mathrm{L}^{2}(\mathbb{R})\right)},
$$

$$
\begin{aligned}
\int_{0}^{T}\|u(t)\|_{\mathrm{L}^{2}(\mathbb{R})}^{2} \mathrm{~d} t & =2 \int_{0}^{T} \Re\left(-i \int_{0}^{t} \Phi h \bar{u} \mathrm{~d} x \mathrm{~d} s\right) \mathrm{d} t \\
& \leq 2 T\|\Phi\|_{c}\|h\|_{\mathrm{L}^{2}\left(0, T ; \mathrm{L}^{2}(\mathbb{R})\right)}\|u\|_{\mathrm{L}^{2}\left(0, T ; \mathrm{L}^{2}(\mathbb{R})\right)}
\end{aligned}
$$

thus,

$$
\|h\|_{\mathrm{L}^{2}\left(0,+\infty ; \mathrm{L}^{2}(\mathbb{R})\right)}^{2} \geq \frac{1-\gamma}{T\|\Phi\|_{c}^{2}} .
$$

Second bound: The boundary conditions $\|u(T)\|_{\mathrm{L}^{2}(\mathbb{R})}^{2}<4(1-\gamma)$ and $\left\|u_{0}\right\|_{\mathrm{L}^{2}(\mathbb{R})}^{2}=4$ give both that along with Cauchy Schwarz inequality

$$
4 \gamma<2\|\Phi\|_{c}\|h\|_{\mathrm{L}^{2}\left(0,+\infty ; \mathrm{L}^{2}(\mathbb{R})\right)}\|u\|_{\mathrm{L}^{2}\left(0, T ; \mathrm{L}^{2}(\mathbb{R})\right)}
$$

and also along with Cauchy Schwarz and integration over time

$$
\|u\|_{\mathrm{L}^{2}\left(0, T ; \mathrm{L}^{2}(\mathbb{R})\right)}^{2}-4 T \leq 2 T\|\Phi\|_{c}\|h\|_{\mathrm{L}^{2}\left(0,+\infty ; \mathrm{L}^{2}(\mathbb{R})\right)}\|u\|_{\mathrm{L}^{2}\left(0, T ; \mathrm{L}^{2}(\mathbb{R})\right)} .
$$

Consequently, it follows that

$$
\|u\|_{\mathrm{L}^{2}\left(0, T ; \mathrm{L}^{2}(\mathbb{R})\right)} \leq T\|\Phi\|_{c}\|h\|_{\mathrm{L}^{2}\left(0, T ; \mathrm{L}^{2}(\mathbb{R})\right)}\left(1+\sqrt{1+\frac{4}{T\|\Phi\|_{c}^{2}\|h\|_{\mathrm{L}^{2}\left(0, T ; \mathrm{L}^{2}(\mathbb{R})\right)}^{2}}}\right) .
$$

Thus, we obtain

$$
\frac{2 \gamma}{T\|\Phi\|_{c}^{2}}<\|h\|_{\mathrm{L}^{2}\left(0,+\infty ; \mathrm{L}^{2}(\mathbb{R})\right)}^{2}\left(1+\sqrt{1+\frac{4}{T\|\Phi\|_{c}^{2}\|h\|_{\mathrm{L}^{2}\left(0, T ; \mathrm{L}^{2}(\mathbb{R})\right)}^{2}}}\right)
$$

and since the function $x \rightarrow x\left(1+\sqrt{1+\frac{4}{x}}\right)$ is increasing on $\mathbb{R}_{+}^{*}$,

$$
\|h\|_{\mathrm{L}^{2}\left(0,+\infty ; \mathrm{L}^{2}(\mathbb{R})\right)}^{2}>\frac{\gamma^{2}}{T\|\Phi\|_{c}^{2}(1+\gamma)^{2}} .
$$

The upper bound follows.

Remark 6.3. The estimates used in the proof of the above result only use the fact that the nonlinearity acts as a potential. Indeed the same result holds for any nonlinearity of this type.

\subsection{Lower bounds}

We prove the following lower bounds.

Proposition 6.4. For every positive $T, \gamma \in(0,1)$ and every sequence of operators $\left(\Phi_{n}\right)_{n \in \mathbb{N}}$ in $\mathcal{L}_{2}^{0,1}$ such that for every $h$ in

$$
\left\{h \in \mathrm{L}^{2}\left(0, T ; \mathrm{L}^{2}(\mathbb{R})\right): h(t, x)=i \frac{\eta^{\prime}(t)}{\eta(t)} \Psi_{\eta}(t, x)-i \sqrt{2} \eta^{\prime}(t) \exp \left(-i \int_{0}^{t} \eta^{2}(s) \mathrm{d} s\right) \eta(t) x \frac{\sinh }{\cosh ^{2}}(\eta(t) x)\right\}
$$

where

$$
\Psi_{\eta}(t, x)=\sqrt{2} \eta(t) \exp \left(-i \int_{0}^{t} \eta^{2}(s) \mathrm{d} s\right) \operatorname{sech}(\eta(t) x)
$$


and $\eta$ is of the following parameterized form where $\tilde{\gamma}$ belongs to a countable dense subset of $(0,1)$

$$
\eta_{\tilde{\gamma}, T}(t)=(2-\tilde{\gamma}-2 \sqrt{1-\tilde{\gamma}})\left(\frac{t}{T}\right)^{2}+2(-1+\sqrt{1-\tilde{\gamma}}) \frac{t}{T}+1,
$$

for almost every $t \in[0, T], \Phi_{n} h(t)$ converges in $\mathrm{H}^{1}(\mathbb{R})$ to $h(t)$ and there exists $C$ positive such that for almost every $t \in[0, T],\left\|\Phi_{n} h(t)\right\|_{\mathrm{H}^{1}(\mathbb{R})} \leq C\|h(t)\|_{\mathrm{H}^{1}(\mathbb{R})}$, we obtain the following inequalities where the $n$ in the error probabilities is there to recall that $\Phi$ is replaced by $\Phi_{n}$

$$
\varliminf_{n \rightarrow \infty, \epsilon \rightarrow 0} \epsilon \log \mathbb{P}_{\epsilon, n}^{\mid 0} \geq-\frac{2(1-\gamma)\left(12+\pi^{2}\right)}{9 T}
$$

and

$$
\varliminf_{n \rightarrow \infty, \epsilon \rightarrow 0} \epsilon \log \mathbb{P}_{\epsilon, n}^{11} \geq-\frac{2(2-\gamma-2 \sqrt{1-\gamma})\left(12+\pi^{2}\right)}{9 T} .
$$

Proof. Consider first that " $\Phi=I "$, denote the corresponding skeletons by $\tilde{\mathbf{S}}_{W N}$ when the initial datum is 0 and by $\mathbf{S}_{W N}$ when it is $\Psi(x)=\sqrt{2} \operatorname{sech}(x)$, they are defined from the $i i /$ of the Strichartz estimates on $\mathrm{L}_{l o c}^{1}\left(0,+\infty ; \mathrm{H}^{1}(\mathbb{R})\right)$, suppose also that $\eta$ is any function of $\mathrm{C}([0, T])$. Since the initial data 0 or $\Psi$ belong to $\mathrm{H}^{2}(\mathbb{R})$, for $h \in \mathrm{L}^{2}(\mathbb{R}), \mathbf{S}_{W N}(h)$ and $\tilde{\mathbf{S}}_{W N}(h)$ are functions of $\mathrm{C}\left([0, T] ; \mathrm{H}^{2}(\mathbb{R})\right) \cap \mathrm{C}^{1}\left([0, T] ; \mathrm{L}^{2}(\mathbb{R})\right)$, consequently $t \rightarrow \eta(t)=\frac{1}{4}\left\|\Psi_{\eta}(t, \cdot)\right\|_{\mathrm{L}^{2}(\mathbb{R})}^{2}$ is necessarily a function in $\mathrm{C}^{1}([0, T])$. Also, for controls $h_{\eta}$ parameterized as in the above assumptions, $\eta$ is in $\mathrm{C}^{1}([0, T])$, the controls belong to $\mathrm{L}_{l o c}^{1}\left(0,+\infty ; \mathrm{H}^{1}(\mathbb{R})\right)$, the skeletons are the prescribed paths $\Psi_{\eta}$ and we obtain

$$
\inf _{\eta \in \mathrm{C}^{1}([0, T]):\left\|\tilde{\mathbf{S}}_{W N}\left(h_{\eta}\right)(T, \cdot)\right\|_{\mathrm{L}^{2}(\mathbb{R})}^{2} \geq 4(1-\gamma)}\left\{\left\|h_{\eta}\right\|_{\mathrm{L}^{2}\left(0,+\infty ; \mathrm{L}^{2}(\mathbb{R})\right)}^{2}\right\}=\inf _{\eta \in \mathrm{C}^{1}([0, T]), b . c .} \int_{0}^{T} F\left(\eta(t), \eta^{\prime}(t)\right) \mathrm{d} t,
$$

where the Lagrangian $F$ is

$$
F(z, p)=\frac{1}{9}\left(12+\pi^{2}\right) \frac{p^{2}}{z}
$$

and b.c. stands for the boundary conditions $\eta(0)=0$ and $\eta(T) \geq 1-\gamma$. Indeed, since $\tilde{\mathbf{S}}_{W N}(h)(T)$ is a function of $(h(t))_{t \in[0, T]}$, the infimum could be taken on functions set to zero almost everywhere after $T$, thus $\|h\|_{\mathrm{L}^{2}\left(0,+\infty ; \mathrm{L}^{2}(\mathbb{R})\right)}^{2}$ in the left hand side could be replaced by $\|h\|_{\mathrm{L}^{2}\left(0, T ; \mathrm{L}^{2}(\mathbb{R})\right)}^{2}$. A scaling argument gives that the terminal boundary condition is necessarily saturated.

Similarly, for the second error probability, $\tilde{\mathbf{S}}_{W N}$ is replaced by $\mathbf{S}_{W N}$ and b.c. is $\eta(0)=1$ and $\eta(T)=1-\gamma$. The usual results of the indirect method do not apply to the problem of the calculus of variations, nonetheless solutions of the boundary value problem associated to the Euler-Lagrange equation

$$
2 \frac{\eta^{\prime \prime}}{\eta}=\left(\frac{\eta^{\prime}}{\eta}\right)^{2}
$$

provide upper bounds when we compute the integral of the Lagrangian. If we suppose that $\eta$ is in $\mathrm{C}^{3}([0, T])$ and that it is positive on $(0, T)$, we obtain by derivation of the ODE that on $(0, T)$,

$$
\eta^{\prime \prime \prime}=0
$$

Also, looking for solutions of the form $a t^{2}+b t+c$, we obtain that necessarily $b^{2}=4 a c$. Thus $\mathrm{C}^{3}([0, T])$ positive solutions are necessarily of the form $a\left(t-\frac{b}{2 a}\right)^{2}$. From the boundary conditions, we obtain that for the first error probability the function defined by

$$
\eta^{0}(t)=(1-\gamma)\left(\frac{t}{T}\right)^{2}
$$


is a solution of the boundary value problem. For the second error probability, the boundary conditions imply that the two following functions defined by

$$
\eta^{1,1}(t)=(2-\gamma+2 \sqrt{1-\gamma})\left(\frac{t}{T}\right)^{2}+2(-1-\sqrt{1-\gamma}) \frac{t}{T}+1
$$

and

$$
\eta^{1,2}(t)=(2-\gamma-2 \sqrt{1-\gamma})\left(\frac{t}{T}\right)^{2}+2(-1+\sqrt{1-\gamma}) \frac{t}{T}+1
$$

are solutions of the boundary value problem. The second function gives the smallest value when we compute the integral of the Lagrangian.

From the assumptions on the operators $\Phi_{n}$ and Lebesgue's dominated convergence theorem along with Hölder's inequality and Lemma 5.2, for functions $h$ of the assumptions of the proposition, $\left(\mathcal{G} \circ \Lambda \circ \Phi_{n}\right) h$ converges to $(\mathcal{G} \circ \Lambda) h=\tilde{\mathbf{S}}_{W N}(h)$ in $\mathrm{C}\left([0, T] ; \mathrm{L}^{2}(\mathbb{R})\right)$. In the above $\mathcal{G}$ is the mapping defined in Section 2.5 with null initial datum. Thus $\left\|\tilde{\mathbf{S}}_{n}(h)(T, .)\right\|_{\mathrm{L}^{2}(\mathbb{R})}^{2}$ converges to $\left\|\tilde{\mathbf{S}}_{W N}(h)(T, .)\right\|_{\mathrm{L}^{2}(\mathbb{R})}^{2}$. As a consequence, for $h$ in the particular parameterized set of controls where $\tilde{\gamma}=\gamma+\delta$ and $\delta>0$, there exists $N_{0}$ large enough such that for any $n \geq N_{0},\left\|\tilde{\mathbf{S}}_{W N}(h)(T, .)\right\|_{L^{2}(\mathbb{R})}^{2} \geq 4(1-\gamma+\delta)$ implies that $\left\|\tilde{\mathbf{S}}_{n}(h)(T, .)\right\|_{L^{2}(\mathbb{R})}^{2}>4(1-\gamma)$. Thus the infimum in the rate for a fixed $\gamma$ is smaller than the infimum on the smallest particular set of controls $h$, which is itself smaller than the square of the $\mathrm{L}^{2}$-norm of the control $h$ corresponding to $\eta_{\gamma, T}$. Indeed $h$ is such that $\left\|\tilde{\mathbf{S}}_{W N}(h)(T, .)\right\|_{\mathrm{L}^{2}(\mathbb{R})}^{2} \geq 4(1-\gamma+\delta)$, for $n$ large enough, which implies the expected boundary condition. We conclude from the upper bound obtained in the previous study of the problem of calculus of the variation by guessing the likeliest path, by taking the limit inf in $n$ of the opposite and from the fact that $\delta$ is then arbitrary. The end of the proof of the lower bound for the second error probability is the same.

We have finally obtained upper and lower bounds that agree up to constants in their behavior in large $T$ for the two error probabilities. In the case of the first error probability they also agree in their behavior in $\gamma$ for $\gamma$ near 1 , it is not quite the case for the second error probability and $\gamma$ near zero. The bounds for the first error probability are of the same order as the one we could obtain from the results of [16]. Indeed, with a slightly different normalization on the NLS equation and when the noise is the ideal white noise and thus $\|\Phi\|_{c}=1$, a result of [16] is that the probability density function of the mass of the pulse at the coordinate $T$ of the fiber when the initial datum is null is asymptotically that of an exponential law of parameter $\frac{\epsilon T}{2}$. Integrating the density over $[2(1-\gamma),+\infty)$, we obtain that $\lim _{\epsilon \rightarrow 0} \epsilon \log \mathbb{P}_{\epsilon}^{\mid 0}=-\frac{4(1-\gamma)}{T}$ which is indeed in between the two bounds and very close to the lower bound though obtained with a more general parametrization. Also in Section 6 of [17], the authors study numerically the second error probability by integrating the joint probability density function over a domain for the soliton power and timing jitter which depends on the size of the window and the threshold. They also consider the unrealistic case where the size of the window is large and of the order of the coordinate $T$ of the location of the receiver in the fiber. The effect of the timing jitter could then be neglected and they obtain an error probability given by $\lim _{\epsilon \rightarrow 0} \epsilon \log \mathbb{P}_{\epsilon}^{\mid 1}=-\frac{c(\gamma)}{T}$, with a constant $c(\gamma)$ which is a function of the threshold. It indeed exhibits the same behavior in $T$ as the one obtained in our previous calculations.

Remark 6.5. In the proposition we would like to impose that the operator $\Phi$ is acting as the identity map on $\operatorname{span}\left\{\frac{1}{\cosh (a x)}, x \frac{\sinh }{\cosh ^{2}}(a x) ; a \in[0,1]\right\}$ but it seems too strong to be compatible with the Hilbert-Schmidt assumption. On the other hand, we may check that the assumptions made here can easily be fulfilled. Also, under these assumptions, the noise is as close as possible to the space-time white noise considered in $[16,17]$ that we are not able to treat mathematically.

Remark 6.6. Note that it is natural to obtain that the opposite of the error probabilities are decreasing functions of $T$. Indeed, the higher is $T$, the less energy is needed to form a signal which mass gets above a fixed threshold at the coordinate $T$. Replacing above by under, we obtain the same result in the case of a soliton 
as initial datum. Consequently, the higher is $T$ the higher the error probabilities get. Also, in the case of the first error probability, both upper and lower bounds in Propositions 6.2 and 6.4 are increasing functions of $\gamma$. Similarly, in the case of the second error probability, the bounds are decreasing functions of $\gamma$. This could be interpreted as the higher is the threshold, the more energy is needed to form a signal which mass gets above the threshold at the coordinate $T$ and conversely in the case of a soliton as initial datum.

Remark 6.7. The results obtained numerically for a parametrization by the amplitude solely without modulating the phase and other shape parameters or of the phase $\eta^{2}(t) t$ instead of $\int_{0}^{t} \eta^{2}(s) \mathrm{d} s$ in (6.1), gave less interesting lower bounds that did not exhibit the desired properties in $T$.

Remark 6.8. In [16] the following parametrization

$$
u(t, x)=\Psi_{p}(t, x)=\sqrt{2} \eta(t) \exp (i \beta(t) x+i \alpha(t)+i \tau(t))[\operatorname{sech}(\eta(t)(x-y(t)))+v(t, x)], \text { with } \tau^{\prime}(t)=\eta^{2}(t)
$$

is suggested. The authors give a physical justification of the fact that for large $T$ the field $v$ could be neglected. This could be compared with the results on asymptotic stability for the deterministic nonlinear Schrödinger equation. They also neglect the parameter $\alpha$. This more complete parametrization has been used to obtain lower bounds of the tails of the soliton center, which is the timing jitter times the mass, of the order of $-\frac{1}{T^{3}}$. It did not require to let the amplitude $\eta$ vary but required the parameters $y, \beta$ and $\alpha$. Upper bounds of the same order in $T$ have been obtained in the case of both an additive and a multiplicative noise considering an equation for the evolution of the soliton center for the controlled equations. This proves that it is way more likely to obtain a shift in the arrival time of the pulse than a decrease of its mass. This result could be restated as the soliton center fluctuates more than the mass. If the law were Gaussian, the variance would be of the order $T^{3}$. This is another theoretical proof of the Gordon-Haus effect. It will appear elsewhere.

Acknowledgements. The author is grateful to the anonymous referee for valuable comments that have led to an improvement of the paper.

\section{REFERENCES}

[1] R. Azencott, Grandes déviations et applications, in École d'été de Probabilité de Saint-Flour, P.L. Hennequin Ed. SpringerVerlag, Berlin. Lect. Notes Math. 774 (1980) 1-176.

[2] A. Badrikian and S. Chevet, Mesures cylindriques, espaces de Wiener et fonctions aléatoires Gaussiennes. Springer-Verlag, Berlin. Lect. Notes Math. 379 (1974).

[3] Y.M. Berezansky, Z.G. Sheftel and G.F. Us, Functional Analysis, Vol. 1. Oper. Theor. Adv. Appl. 85 (1997) 125-134.

[4] G. Buttazzo, M. Giaquinta and S. Hildebrandt, One-dimensional Variational Problems. Oxford University Press, Oxford. Oxford Lect. Ser. Math. Appl. 15 (1998).

[5] T. Cazenave, An Introduction to Nonlinear Schrödinger Equations. Instituto de Matématica-UFRJ Rio de Janeiro, Brazil. Textos de Métodos Matématicos 26 (1993).

[6] G. Da Prato and J. Zabczyk, Stochastic Equations in Infinite Dimensions. Cambridge University Press: Cambridge, England. Encyclopedia Math. Appl. (1992).

[7] A. de Bouard and A. Debussche, The Stochastic Nonlinear Schrödinger Equation in $\mathrm{H}^{1}$. Stochastic Anal. Appl. 21 (2003) $97-126$.

[8] A. de Bouard and A. Debussche, On the effect of a noise on the solutions of the focusing supercritical nonlinear Schrödinger equation. Probab. Theory Relat. Fields 123 (2002) 76-96.

[9] A. de Bouard and A. Debussche, Finite time blow-up in the additive supercritical nonlinear Schrödinger equation: the real noise case. Contemp. Math. 301 (2002) 183-194.

[10] A. Debussche and L. Di Menza, Numerical simulation of focusing stochastic nonlinear Schrödinger equations. Phys. D 162 (2002) 131-154.

[11] S.A. Derevyanko, S.K. Turitsyn and D.A. Yakusev, Non-gaussian statistics of an optical soliton in the presence of amplified spontaneaous emission. Optics Lett. 28 (2003) 2097-2099.

[12] J.D. Deuschel and D.W. Stroock, Large Deviations. Academic Press, New York. Pure Appl. Math. (1986).

[13] A. Dembo and O. Zeitouni, Large deviation techniques and applications (2nd edition). Springer-Verlag, New York. Appl. Math. 38 (1998). 
[14] P.D. Drummond and J.F. Corney, Quantum noise in optical fibers. II. Raman jitter in soliton communications. J. Opt. Soc. Am. B 18 (2001) 153-161.

[15] L.C. Evans, Partial Differential Equations. American Mathematical Society, Providence, Rhode Island, Grad. Stud. in Math. 119 (1998).

[16] G.E. Falkovich, I. Kolokolov, V. Lebedev and S.K. Turitsyn, Statistics of soliton-bearing systems with additive noise. Phys. Rev. E 63 (2001) 025601(R).

[17] G. Falkovich, I. Kolokolov, V. Lebedev, V. Mezentsev and S.K. Turitsyn, Non-Gaussian error probability in optical soliton transmission. Physica D 195 (2004) 1-28.

[18] É. Gautier, Uniform large deviations for the nonlinear Schrödinger equation with multiplicative noise. Preprint IRMAR, Rennes (2004). Submitted for publication.

[19] T. Kato, On Nonlinear Schrödinger Equation. Ann. Inst. H. Poincaré, Phys. Théor. 46 (1987) $113-129$.

[20] V. Konotop and L. Vázquez, Nonlinear random waves. World Scientific Publishing Co., Inc.: River Edge, New Jersey (1994).

[21] R.O. Moore, G. Biondini and W.L. Kath, Importance sampling for noise-induced amplitude and timing jitter in soliton transmission systems. Optics Lett. 28 (2003) 105-107.

[22] C. Sulem and P.L. Sulem, The Nonlinear Schrödinger Equation, Self-Focusing and Wave Collapse. Springer-Verlag, New York, Appl. Math. Sci. (1999).

[23] J.B. Walsh, An introduction to stochastic partial differential equations, in École d'été de Probabilité de Saint-Flour, P.L. Hennequin Ed. Springer-Verlag, Berlin, Lect. Notes Math. 1180 (1986) 265-439. 